\title{
1 \\ Introduction to the Solid State - Physical Properties and Processes
}

\author{
Ingvar Ymén \\ AstraZeneca $R \& D$, Södertälje, Sweden
}

\subsection{Introduction}

Pharmaceutical substances consist of organic molecules alone, or in combination with metal cations (metal salts), organic cations, organic or inorganic anions, neutral organic molecules (co-crystals) or solvent molecules (solvates). Even though some are salts, these have little resemblance with inorganic salts, the typical properties of which include high melting points, high hardness and strong ionic bonding. In fact, pharmaceutical substances, as salts or not, generally have similar properties, which are dominated by the presence of the organic molecules and their typical, weak intermolecular interactions. Crystals formed by organic substances are usually referred to as molecular crystals (Kitaigorodsky 1973) and it is the properties of this type of molecules that will be referred to when talking about the properties of pharmaceutical substances. In the following the physical states of a pure pharmaceutical substance will be discussed with reference to Figure 1.1 where the approximate areas of existence as a function of temperature are shown.

\subsubsection{The Gas/Vapour and Liquid States}

A gas consists of molecules, moving independently of each other, but occasionally colliding with each other or with the vessel in which it is contained. The collisions make up the gas pressure and the more collisions the higher is the pressure. A gas containing small, point-like molecules with negligible molecular interactions is called ideal. For such a gas:

$$
\mathrm{pV}=\mathrm{nRT}
$$

Solid State Characterization of Pharmaceuticals, First Edition. Edited by Richard A. Storey and Ingvar Ymén. (C) 2011 Blackwell Publishing Ltd. Published 2011 by Blackwell Publishing Ltd. 


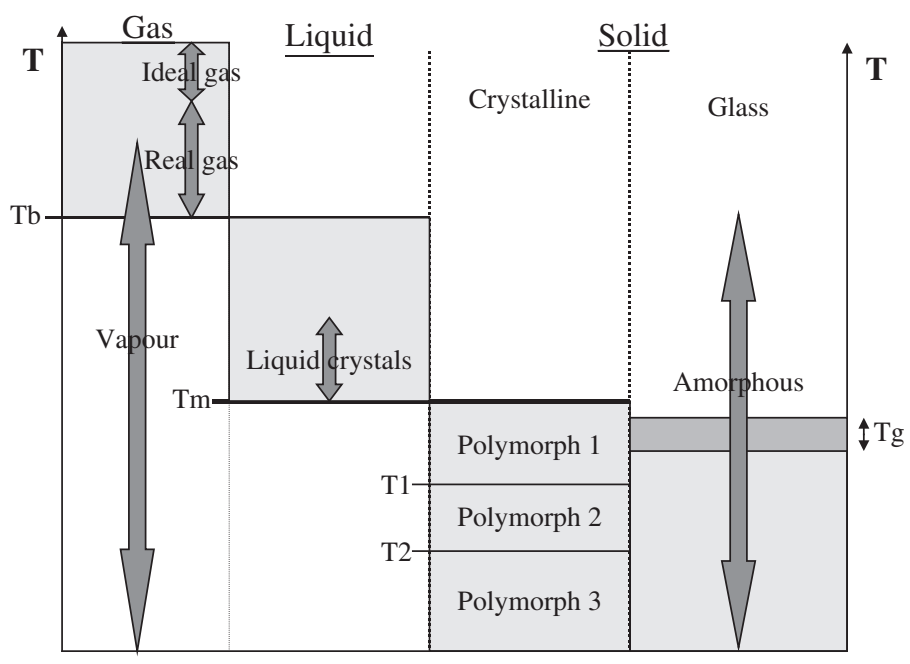

Figure 1.1 Different possible physical states of a pharmaceutical substance as a function of temperature; it is assumed that the pressure is constant at 1 atmosphere

where $\mathrm{p}=$ pressure, $\mathrm{V}=$ volume, $\mathrm{n}=$ the number of moles, $\mathrm{T}=$ temperature and $\mathrm{R}=$ the gas constant. Most gases are nonideal because their molecules are not pointlike and they interact to some extent. Gases at high temperatures are closest to ideal and the deviation from ideality increases closer to the point of condensation. In a mixture of ideal gases each gas will exert a pressure, which is proportional to the amount of that gas. The total pressure is the sum of the individual pressures so that for $\mathrm{n}$ different ideal gas molecules:

$$
\mathrm{p}_{\text {tot }}=\mathrm{p}_{1}+\mathrm{p}_{2}+\cdots+\mathrm{p}_{\mathrm{n}}
$$

In a gas each molecule may interact with itself in an intramolecular way, thus forming various conformations. If the temperature of the gas is close to the condensation point, a certain conformation may be dominating but as the temperature of the gas increases the molecular vibrations and rotations increase. This leads to an increased number of conformations becoming available. Molecular structures of reasonably complex gaseous molecules are usually determined by means of gas-phase electron diffraction (Rankin and Robinson 1995) or microwave spectroscopy (Consalvo and Stahl 1998).

There can only be one gas phase, regardless of the number of different molecular species in it. Such a gas phase will be completely isotropic (have the same physical properties in all directions), have a very low density, low viscosity and a high compressibility.

Lowering the temperature, the gas molecules will gradually lose translational and vibrational/rotational energy. At a certain temperature, the condensation/boiling point, the molecules will, when colliding, start forming aggregates and a liquid will form. The temperature at which this occurs, depends on the size of the molecule and on the number and strength of the bonds formed. Small molecules, forming only weak intermolecular interactions, will have low boiling points whereas larger molecules, forming stronger, or a larger number of, intermolecular bonds have higher boiling points. It should be noted that the condensation process depends on seeds on which the drops of liquid can form. In the absence of seeds the vapour may be supercooled.

Upon condensation the entropy decreases and a latent enthalpy of condensation is given off, thus lowering the total energy of the liquid. The molecules in the liquid will, however, still have 
energy enough to perform translational motion, but not enough to escape molecular interactions. Consequently molecular movement will no longer be independent. There will also be a tendency towards fewer molecular conformations and towards the formation of larger molecular entities, such as dimers. For this reason the molecules themselves are no longer completely randomly oriented. This short-range order in liquids can be analysed with small angle X-ray diffraction, SAXS (Klug and Alexander, 1974) and the presence of dimers and larger aggregates can be determined by spectroscopic methods (Spitaleri et al. 2004)). Still, the lack of long-range order makes all physical properties of liquids isotropic. These properties are, however, distinctly different from those of the gas.

A liquid at ambient conditions will establish equilibrium with its own gas phase, referred to as the liquids vapour (this word is sometimes also used to describe gases close to the condensation point). The pressure, which is a function of temperature, is called the vapour pressure. The maximum amount of vapour in equilibrium with liquid water at a certain temperature is called the saturation vapour pressure, $\mathrm{p}_{\mathrm{sat}}$. This pressure increases with increasing temperature and for water it becomes $101 \mathrm{kPa}$ at $100^{\circ} \mathrm{C}$, the boiling point of water. Dividing the actual water vapour pressure, $\mathrm{p}_{\mathrm{H}_{2} \mathrm{O}}$ at a certain temperature with the saturation vapour pressure at the same temperature yields the relative humidity or the \% RH:

$$
\% \mathrm{RH}=100 *\left(\mathrm{pH}_{2} \mathrm{O} / \mathrm{p}_{\mathrm{sat}}\right) .
$$

To avoid confusion one must always relate the $\% \mathrm{RH}$ to temperature. The measurement of water vapour pressure in ambient air, $\mathrm{p}_{\mathrm{H}_{2} \mathrm{O}}$ is usually done as a dew point measurement (Moss 1934). An important difference between liquid and gas is that different liquids, when brought together, need not mix. Thus multiple liquid phases may be observed together, for instance in emulsions.

\subsubsection{The Crystalline State}

As a liquid is cooled the molecular movements decrease. Depending on the rate of cooling and on the properties of the liquid, either crystallization or glass formation will occur. For small, simple molecules at low cooling rates crystallization will normally occur at or below the freezing/melting point. There the molecules start forming intermolecular bonds, which are strong enough to stop translational, but not vibrational/rotational, motion. The freezing/melting point, like the boiling point, will be higher for larger molecules and for molecules with more and stronger interactions.

Upon crystallization the entropy decreases and a latent enthalpy of crystallization is released, which may be recorded as an exothermic event with, for instance, a DSC. Since crystallization is always more or less supercooled, an accurate determination of the theoretical onset temperature of crystallization (the freezing point) must instead be performed as a melting point analysis. The degree of super-cooling of the freezing point depends on molecular complexity and on presence of seeds.

The main similarity between the liquid and crystalline states is that, because the intermolecular distances are similar, the number of molecules per unit volume is roughly the same. This gives crystalline material numerical values of some physical properties, which are in a similar range to those of the liquid, for instance density and compressibility.

The big difference between liquids and crystals is the lack of translational motion and the presence of long-range order in crystals. In a crystal each molecule, with some exceptions (Dunitz and Gavezzotti 2005), has the same conformation and is fixed in one of a few possible, symmetryrelated, positions. In this position, the molecule is bonded to its neighbours via weak intermolecular bonds (for example, hydrogen bonds or van der Waals bonds). In this way a crystal structure, which defines many of the physical properties, is formed.

The crystal structure may be described as a three-dimensional brick structure, built up of identical units cells, each of which is uniquely defined by its three unit cell axes a, b and c and the three 


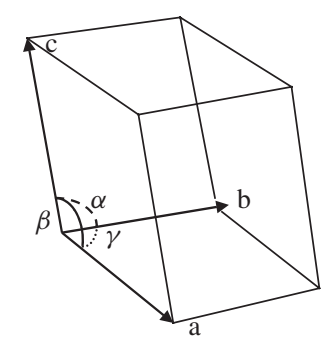

Figure 1.2 The unit cell with unit cell axes and angles indicated

Table 1.1 The seven crystal systems

\begin{tabular}{llllccc}
\hline Crystal system & $\mathrm{a}$ & $\mathrm{b}$ & $\mathrm{c}$ & $\alpha$ & $\beta$ & $\gamma$ \\
\hline Triclinic & $\mathrm{a}$ & $\mathrm{b}$ & $\mathrm{c}$ & $\neq 90^{*}$ & $\neq 90^{*}$ & $\neq 90^{*}$ \\
Monoclinic & $\mathrm{a}$ & $\mathrm{b}$ & $\mathrm{c}$ & 90 & $\neq 90$ & 90 \\
Orthorhombic & $\mathrm{a}$ & $\mathrm{b}$ & $\mathrm{c}$ & 90 & 90 & 90 \\
Tetragonal & $\mathrm{a}$ & $\mathrm{b}$ & $\mathrm{a}$ & 90 & 90 & 90 \\
Trigonal & $\mathrm{a}$ & $\mathrm{a}$ & $\mathrm{a}$ & $\neq 90^{*}$ & $\neq 90^{*}$ & $\neq 90^{*}$ \\
Hexagonal & $\mathrm{a}$ & $\mathrm{b}$ & $\mathrm{a}$ & 90 & 120 & 90 \\
Cubic & $\mathrm{a}$ & $\mathrm{a}$ & $\mathrm{a}$ & 90 & 90 & 90 \\
\hline
\end{tabular}

*In the trigonal system all three angles are the same but in the triclinic they are all different.

angles $\alpha, \beta$, and $\gamma$, between these axes (Figure 1.2). The combination of these axes and angles gives rise to seven possible crystal systems (Table 1.1). Any crystal structure can be described with a unit cell from one of the crystal systems.

The unit cell usually contains more than one molecule, and these molecules, if having the same conformation, are related to each other via symmetry operations, described by the space group (Giacovazzo 1992). The smallest part of the unit cell in which there is no symmetry is called the asymmetric unit (often only one molecule). The number of asymmetric units in the unit cell is denominated as $\mathrm{Z}$ and it is basically the number of different molecular conformations present in the crystals structure. There is a relation between $\mathrm{Z}$, the unit cell volume $\mathrm{V}$, the molecular weight $\mathrm{M}_{\mathrm{w}}$, Avogadro's number $\mathrm{N}_{\mathrm{A}}$ and the density d:

$$
\mathrm{d}=\mathrm{ZM}_{\mathrm{w}} / \mathrm{VN}_{\mathrm{A}}
$$

With this, densities are accurately calculated or, with an accurate density from, for example, pycnometry (Tamari and Aquilar-Chávez 2004) and a unit cell from X- ray data, the molecular weight can be checked.

In a crystal structure the molecules are packed efficiently and once the crystal structure is determined the packing efficiency can be calculated as the amount of filled space in the unit cell divided by the total unit cell volume. The value obtained is called the packing coefficient, which for organic molecules usually is in the range 0.65-0.73 (Kitaigorodsky 1973). Packing coefficients below 0.6 are rarely encountered.

Historically crystallography relied a lot on microscopy but today more than $99 \%$ is performed using X-rays together with either single crystals (SXRD) or a microcrystalline powder (XRPD). Table 1.2 lists a number of investigations, which can be performed by means of crystallography.

Both the crystal habit and the fracture of crystals are closely related to the crystal structure. Directions in the crystal structure where intermolecular bonding is weak will give rise to long unit cell axes and directions of stronger bonding give shorter axes. The crystal growth rate will 
Table 1.2 Typical crystallographic information obtained from single crystal X-ray diffraction (SXRD), $X$-ray powder diffraction (XRPD) and polarized light microscopy (PLM). Figures indicate which method is most suitable

\begin{tabular}{|c|c|c|c|}
\hline Information searched & SXRD & XRPD & PLM \\
\hline Is the sample crystalline or amorphous? & $3^{*}$ & 1 & 2 \\
\hline What is the degree of crystallinity of the sample? & & 1 & \\
\hline Fingerprint identity of a single phase & $3^{*}$ & 1 & 3 \\
\hline Is the sample a single phase? & $3^{*}$ & 1 & 1 \\
\hline Quantify the amounts of several phases in a mixture & & 1 & \\
\hline Determination of crystal structure & 1 & 2 & \\
\hline Unit cell data & $1^{* *}$ & $1^{* *}$ & \\
\hline Optical data & & & 1 \\
\hline
\end{tabular}

* SXRD uses one or a few single-crystals and will thus not give relevant information for larger microcrystalline samples. ** It is usually easier to determine the unit cell using SXRD but XRPD gives a higher accuracy of the unit cell parameters. Therefore XRPD is often used to refine unit cell data obtained using SXRD.

be higher in the direction of stronger bonds, so a needle-shaped crystal will usually have the strongest bonds (a short unit cell axis) in the needle direction. Similarly, a very thin plate shaped crystal will have a long unit cell axis (weakest bonding direction) perpendicular to the plane of the plate. Likewise, when forces are applied on crystals they are likely to fracture along directions of weakest bonding, classical examples of which are talcum and graphite.

Control of crystal habit and of crystal size is of great importance, because these may have a large impact on material processing such as filtration, transport (flow properties), tableting, granulation and so forth. The most common ways to affect the crystal growth and habit are by choice of solvent and of supersaturation level for the crystallization. Moreover, because small amounts of foreign molecules may also have a large (usually negative) impact on both size and habit of crystals, it is common practice to purify materials as much as possible prior to crystallization. However, if done in a controlled way, the addition of small amounts of carefully selected substances may be beneficial both for habit modification and crystal size (Meenan et al. 2002).

The optical properties of crystals are also strongly related to the crystal structure and is more discussed by Bunn (1948).

\subsubsection{The Glassy State}

As noted above, depending on the rate of cooling and on the properties of the liquid, either crystallization or glass formation will occur. If a liquid containing comparably large and complex molecules, is cooled at a high rate the onset of crystallization might be severely overshot, resulting in the formation of a super-cooled liquid. Such a liquid will have a high viscosity, which upon continuous cooling eventually will become so high, that translational molecular movement will almost stop, at least compared to the time-scale of normal experiments. At this point the viscosity will be roughly $10^{13}$ poise (Ediger et al. 1996) and a glass has formed.

A glass is often referred to as an amorphous material, which is somewhat ambiguous if one wants to distinguish it from a normal or a supercooled liquid. All three types of materials are in fact amorphous, meaning that they all lack the long-range order found in crystals. A comparison of the three by means of XRPD analysis shows that they all lack sharp peaks. Although lacking longrange order, amorphous materials usually have some short-range ordering such as the formation of hydrogen-bonded dimers, trimers and so forth in solutions of carboxylic acids.

Materials that are partially crystalline are sometimes observed. In XRPD-diffractograms this is indicated by an increased background level in some regions an/or as peak broadening (Klug and Alexander 1974). Partial crystallinity may be described by a one-phase model or a two-phase 
model (Westdahl 1996), where the one-phase model assumes disorder in the crystalline material and the two-phase model assumes that the sample is a mixture of two phases, one completely crystalline and one completely amorphous. Which method is preferred depends on what type of material one is working with. For pharmaceutical substances the latter model is normally used. The degree of crystallinity may then be analysed with various techniques (Saleki-Gerhardt et al. 1994; Buckton and Darcy 1999).

Along the cooling process, from the melting point until the point of glass formation, all physical properties of the super-cooled liquid show the same gradual change upon cooling, as they do for the melt above the melting point. At the point of glass formation, the molecules have come so close together that this gradual change can no longer continue. Instead, upon continued cooling a different path is now followed so a new type of material has indeed appeared. The most common physical properties used to describe glass formation are the specific heat $C_{p}$ or the specific volume $\mathrm{V}_{\mathrm{sp}}$, both of which show distinct changes when the glass forms (Figures 1.3 and 1.4).

The point of glass formation is called the glass transition temperature, $T_{g}$. Despite its name, it does not signify a true phase transition. Its value is not a constant but varies with the rate of cooling, so that the lower the rate, the lower the glass transition temperature and the lower the $C_{p}$ and the $\mathrm{V}_{\mathrm{sp}}$. This is a consequence of the fact that at a slower rate the molecules have more time to pack more efficiently.

The glass transition is usually analysed using DSC and most conveniently using the modulated type. With a hot stage microscope, in heating mode, $\mathrm{T}_{\mathrm{g}}$ can also be seen as the morphological change from a chip-like solid to a rounded liquid drop.

The glass transition temperature can also be affected by impurities and will in many cases drop significantly by the uptake of water. This is the basis for the determination of the amount of

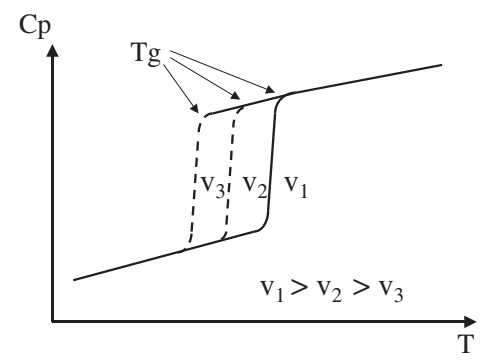

Figure 1.3 Variation of $C_{p}$ as a function of temperature for a liquid that becomes a glass; the effect of different cooling-rates $v 1, v 2$ and $v 3$, is also indicated

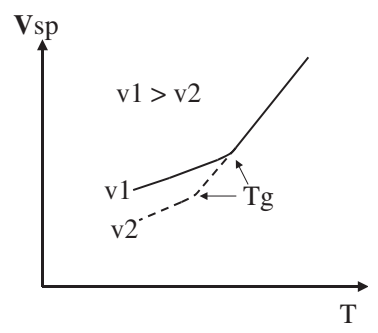

Figure 1.4 Variation of $V_{s p}$ as a function of temperature for a liquid that becomes a glass; the effect of different cooling-rates $v 1$ and $v 2$, is also indicated 
glass in a sample, using isothermal calorimeters (Ahlneck et al. 1994). The material is allowed to take up water so that $\mathrm{T}_{\mathrm{g}}$ gradually drops. Crystallization occurs when it reaches the temperature of the calorimeter. The heat of crystallization is proportional to the amount of glass present in the sample. Since grinding of pharmaceutical substances often destroys parts of the crystal structure, amorphous material may be formed upon milling or grinding. The isothermal calorimeter measurements described above works very well for the quantification of the amount of amorphous material thus formed (Buckton et al. 1994).

The best analyses for the identification of a glass are polarized light microscopy (indicates optically isotropic behaviour), XRPD (no peaks) and DSC (shows $\mathrm{T}_{\mathrm{g}}$ ).

Other methods, such as isothermal calorimetry, moisture sorption and spectroscopic methods, will not offer identification on their own but they are good complimentary methods.

\subsection{Neutral Pharmaceutical Molecules}

Figure 1.5 shows a typical hypothetical, neutral pharmaceutical molecule in which different parts or groups are circled. What parts are present and how these are combined will determine the molecular properties and at the macroscopic level also the physical properties of the substance. Some fundamental molecular properties of importance from the solid-state perspective will be discussed below.

In a pharmaceutical molecule the groups with the lowest or highest pKa values can be exploited when preparing salts. The molecule in Figure 1.5 has a typically acidic group, a carboxylic acid and a typically alkaline group, a secondary amine. These groups will be responsible for the main protolytic properties of the molecule - that is, they will have the highest and the lowest $\mathrm{pK}_{\mathrm{A}}$ values. In fact, a substance like this, having both an acidic and an alkaline group, may establish an internal acid - base equilibrium and thus behave as a zwitterions in the $\mathrm{pH}$ range between the two $\mathrm{pK}_{\mathrm{A}}$-values.

The $\mathrm{pK}_{\mathrm{A}}$ value of an isolated functional group is usually easy to predict from the approximate ranges given in an organic chemistry textbook. However, when neighbouring groups interact, by means of resonance, inductive effects and so forth, things become more difficult. Database computer programs may be used to assist but, to be on the safe side, experimental $\mathrm{pK}_{\mathrm{A}}$-determinations should be made by acid-base titrations or NMR analysis (Kubal et al. 1994). It should be noted that $\mathrm{pK}_{\mathrm{A}}$-values, which are usually measured in water solutions, can also be determined in nonaqueous solvents, wherein they will take different values (Barcza and Buvári-Barcza 2003).

Some pharmaceutical molecules are very soluble in water but not in oil whereas others behave in more-or-less the opposite way. This type of behaviour is expressed by means of the partition coefficient between oil and water, $\mathrm{K}_{\mathrm{p}}$, which measures the molecules' approximate affinity for

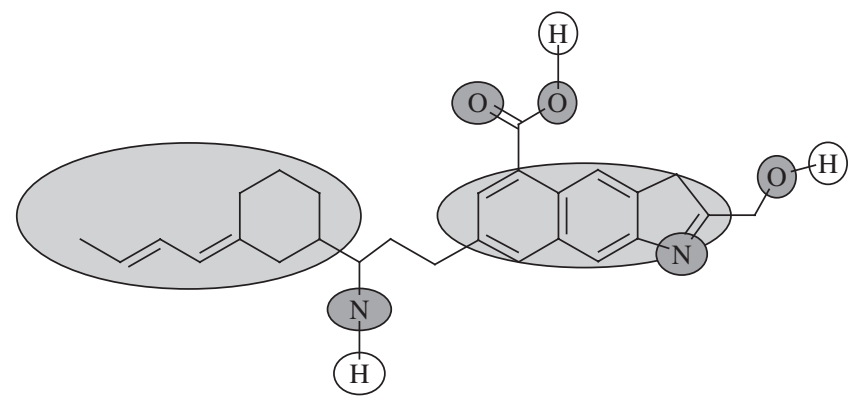

Figure 1.5 A hypothetical pharmaceutical molecule with various functionalities marked 
water and for oil. A molecule with a high affinity for water is referred to as hydrophilic and one with a high affinity for oil as hydrophobic. On a molecular level the main difference between water and oil is the dipole moments of the molecules, that of water being quite high and that of a typical molecule in oil being low. This is the main reason for the high dielectricity constant of water compared to oil. Since most oils almost entirely consist of hydrocarbons it should be clear that hydrocarbon groups in a molecule are hydrophobic. A typical such part is circled in the leftmost side of Figure 1.5.

The parts of the molecule, which have local, high dipole moments, that is, groups in which there are local large charge separations, will be hydrophilic. Such areas are marked with dark circles in Figure 1.5. The fact that the molecule has one hydrophobic and one hydrophilic end means that it is very likely to have surface-active properties. At the interface between a hydrophilic and a hydrophobic medium, it will thus orient itself with its hydrophobic end towards the hydrophobic medium and the hydrophilic end towards the hydrophilic.

The number, size and position of the various hydrophilic and hydrophobic areas of a molecule will be responsible for 'the molecular part' of the solubility of a crystalline substance in a certain solvent (Yalkowsky and Banerjee 1992). This part mainly accounts for how well the molecules fit in and interact with the solvent molecules and is related to the partition coefficient. The other part, which determines the solubility of a substance is 'the crystal structure part', defined by how well the molecules interact in a crystal structure. This part has a strong relation to the melting point. Computer programs aiming to predict solubility solely from the knowledge about molecular structure have so far, for this reason, had limited success.

The density of pharmaceutical substances depends on the molecular density (molecular weight/ molecular volume) and on how well the molecules pack in the solid (see packing coefficient in 1.4). The molecular density is usually the same for substances containing only $\mathrm{C}, \mathrm{H}, \mathrm{N}$ and $\mathrm{O}$, the crystal density of which usually are between $1.2-1.4 \mathrm{~kg} / \mathrm{l}$. With the presence of heavier elements such as $\mathrm{Si}, \mathrm{S}, \mathrm{P}$ and halogens the molecular and crystal density typically increase.

The lowest energy conformation of a hypothetical molecule in a state without any interactions with other molecules can be calculated by means of quantum mechanics 'in vacuum' (Leach 2001). This conformation will be a consequence of the molecule's ambition to lower its total energy by minimizing steric effects and optimizing intramolecular bonding. Steric effects may be overcome if the molecule has enough flexibility, that is, low energy rotational bonds. From the bonding perspective the molecule strives to maximize the number and strength of the bonds formed. The formation of strong intramolecular hydrogen bonds may complicate the molecular analysis. These form between hydrogen bond donors (groups containing circled hydrogen atoms in Figure 1.5) and hydrogen bond acceptors (circled $\mathrm{O}$ - and $\mathrm{N}$-atoms in the figure). If the molecule is large, folding so as to maximize van der Waals contacts might also become very important, as will interactions involving $\pi$-systems. The central aromatic system in Figure 1.5 will be quite inflexible and essentially planar. Quite possibly, in vacuum the terminal unsaturated hydrocarbon chain would fold over the central aromatic ring system so as to optimize $\pi-\pi$ interactions.

Today, quantum chemical modelling may also involve solvent, explicitly or implicitly, allowing more adequate data to be compared to experimental results in solution. However, in many cases theoretical models may fail to properly represent real liquid or solid pharmaceutical systems.

The absorption of visible and ultraviolet light in neutral pharmaceutical molecules is due to the presence of 'chromophores'. These are unsaturated parts of molecules containing multiple bonds. In Figure 1.5 there are many parts that act as chromophores, the unsaturated carbohydrate chain, the central aromatic ring system and the carboxylic acid. Coloured solid pharmaceutical substances usually contain large chromophore systems, in which there are many possible resonance structures. 


\subsection{Thermodynamics and Phase Diagrams}

In order to define the equilibrium state of a chemical system containing a pharmaceutical substance, four basic variables have to be defined, the amount of the substance, the pressure, the temperature and the volume. Since pressure, temperature and volume are always related (for instance, for one mole of an ideal gas $\mathrm{pV}=\mathrm{RT}$ ) only two of these needs to be defined in order to determine the third. On the molecular level pressure is the sum of the forces due to molecular collisions with the container walls and temperature reflects the sum of the kinetic motions of the molecules. Per definition the kinetic energy is thus zero at $\mathrm{T}=0 \mathrm{~K}$.

The identity of the substance is important in order to define the concept of heat. If one mole of a certain substance at $\mathrm{T}=0 \mathrm{~K}$ is allowed to absorb energy the molecules and their constituting atoms will start to move. If a temperature rise of $1 \mathrm{~K}$ is registered, the substance has required an amount of heat, defined by the heat capacity $\mathrm{C}$ of the substance (in units of $\mathrm{J} /\left(\mathrm{mole}_{*}\right.$ degree) ) to be

$$
\mathrm{dq}=\mathrm{CdT}
$$

Different substances have different heat capacities but the heat capacity is not only related to the identity of the substance but also to what state this substance is in. Thus, the heat capacity for a certain substance is not a constant but depends on $\mathrm{p}, \mathrm{T}$ and $\mathrm{V}$. The heat capacity at constant pressure $C_{p}$ and at constant volume $C_{v}$ is quite different for gases but usually differs only slightly for condensed matter.

The heat absorbed or liberated when the reaction proceeds at constant pressure is defined as the enthalpy,

$$
\Delta \mathrm{H}=\mathrm{q}_{\mathrm{p}} .
$$

The heat capacity at constant pressure is related to the enthalpy via

$$
\mathrm{C}_{\mathrm{p}}=(\delta \mathrm{H} / \delta \mathrm{T})_{\mathrm{p}}
$$

where $\delta$ symbolizes a partial derivative. Enthalpies are determined in 'open' (constant pressure) calorimeters.

It should be noted that the experimentally determined values of certain thermodynamic quantities (enthalpy, entropy, free energy etc) for a reaction, are differences before and after a reaction. In order to obtain absolute values a reference state needs to be defined. Depending on the situation, various reference states may be chosen. The enthalpies of all elements have, by convention, been set to zero at $298.15 \mathrm{~K}$ and $1 \mathrm{~atm}$ pressure (the 'standard state'). The standard enthalpy of formation of a chemical substance is thus the enthalpy obtained when the substance is formed from its constituent elements at $298.15 \mathrm{~K}$ and $1 \mathrm{~atm}$.

The temperature dependence of the enthalpy is expressed by Kirchoff's law

$$
\mathrm{d}(\Delta \mathrm{H}) / \mathrm{dT}=\Delta \mathrm{Cp} .
$$

As $\Delta \mathrm{Cp}$ is fairly constant over comparably large temperature intervals it can be used to calculate/estimate enthalpies at temperatures where no experimental data exist.

On the molecular level enthalpy is a measure of the bonding state within and between the molecules in a gas, liquid or solid. For a solid, as a result of the increasing unit cell volume with temperature, and the thereby weaker chemical bonds, the enthalpy increases continuously with temperature. At phase transitions, discontinuous jumps appear, called latent heats of transition. The enthalpy increase at these points reflects the weakening or breaking of bonds during the transition. 
Due to the great importance of spontaneous reactions and equilibrium, the energy type most frequently used in chemical thermodynamics is Gibbs free energy G, which for an isothermal process from state 1 to state 2 is defined by

$$
\Delta \mathrm{G}=\mathrm{G}_{2}-\mathrm{G}_{1}=\Delta \mathrm{H}-\mathrm{T} \Delta \mathrm{S}
$$

This equation summarizes the two main factors driving a system towards equilibrium, the tendency to minimize enthalpy and to maximize entropy. A nonequilibrium system at constant $p$ and $\mathrm{T}$ will only change spontaneously towards equilibrium if the reaction leads to a negative change in Gibbs free energy. Thus, for a spontaneous reaction $\Delta \mathrm{G}<0$ whereas at equilibrium $\Delta \mathrm{G}=0$.

The entropy $\Delta \mathrm{S}$ (in units $\mathrm{J} /$ degree) is a measure of randomness in the system and it is per definition supposed to be zero at $0 \mathrm{~K}$. The entropy increases with increasing randomness. For a phase transition occurring at constant $\mathrm{p}$ and $\mathrm{T}$, where $\Delta \mathrm{G}=0$, then

$$
\Delta \mathrm{S}=\Delta \mathrm{H} / \mathrm{T}
$$

This means that during the phase transition from the low temperature phase to the high temperature phase the heat uptake (increase in enthalpy) is always exactly compensated for by an increase in entropy. On the molecular level an increase in entropy is similar to an increased number of accessible energy states (randomness) of the system, thus entropy increases with temperature. The dynamic disorder (the thermal motion of the atoms) and the static disorder (the number of molecules in 'erroneous' lattice positions) are the main contributors to the total entropy.

$\mathrm{G}$ decreases as the temperature increases. Obviously, $\mathrm{G}$ being a function of $\mathrm{S}$ and $\mathrm{H}$, the $\mathrm{T} \Delta \mathrm{S}$ term grows faster than the $\Delta \mathrm{H}$-term. The temperature dependence of $\mathrm{G}, \mathrm{H}$ and $\mathrm{S}$ are sometimes summarized in a single diagram as shown in Figure 1.6. Using various types of approximations such diagrams have been determined experimentally (Grunenberg et al. 1996; Yu et al. 2000) in order to summarize relations between polymorphs and melts of pharmaceutical substances.

It should be noted that G, H and S are state functions - that is they only depend on the state of the system and their values for a certain state are the same, by whatever path the state has been reached.

For an open system, one to which it is possible to add substance, the change in Gibbs free energy upon addition of $\mathrm{n}$ moles of the chemical species $\boldsymbol{j}$, while keeping all other amounts as well as $\mathrm{p}$ and $\mathrm{T}$ constant, is called the chemical potential

$$
\mu_{j}=\left(\delta \mathrm{G} / \delta \mathrm{n}_{j}\right)_{\mathrm{nj}, \mathrm{p}, \mathrm{T}}, \text { and } \mathrm{G}=\mathrm{n}_{j}{ }^{*} \mu_{j}
$$

For an ideal gas changing from a reference state to another state $B$ with pressures $p_{o}$ and $p_{B}$ it can be show that

$$
\mu=\mu^{\mathrm{o}}+\mathrm{RT} \ln \mathrm{p}_{\mathrm{B}} / \mathrm{p}_{\mathrm{o}}
$$

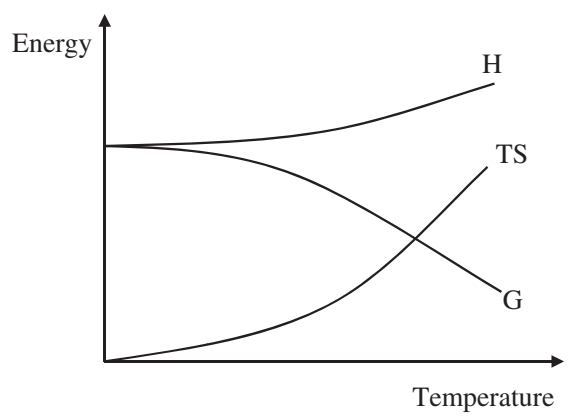

Figure 1.6 The temperature dependence of $G, H$ and TS 
If the gas is not ideal $p_{B}$ has to be exchanged for the fugacity $f_{B}$, which takes into account the deviation from ideality (Ramshaw 1995). The quota $f_{A} / p_{o}$ is called the activity of the real gas so that

$$
\mu=\mu^{\mathrm{o}}+\mathrm{RT} \ln \mathrm{f}_{\mathrm{B}} / \mathrm{p}_{\mathrm{o}}=\mu^{\mathrm{o}}+\mathrm{RT} \ln \mathrm{a} .
$$

As the reference state is normally chosen one where $\mathrm{p}_{\mathrm{o}}=1 \mathrm{~atm}$. A similar derivation may be done for liquids, solids and solutions (Ramshaw 1995). For a general chemical reaction $\mathrm{mX} \leftrightarrows$ $n Y$ then

$$
\Delta \mathrm{G}=\Delta \mathrm{G}^{\mathrm{o}}+\mathrm{RT} \ln \mathrm{Q}, \quad \text { where } \mathrm{Q}=\left[\left(\mathrm{a}_{\mathrm{Y}}\right)^{\mathrm{n}} /\left(\mathrm{a}_{\mathrm{X}}\right)^{\mathrm{m}}\right] .
$$

At equilibrium this becomes $\Delta \mathrm{G}^{\mathrm{o}}=-\mathrm{RT} \ln \mathrm{K}$, where $\mathrm{K}$ is the equilibrium constant that contains the activities of the reactants and the products. The activities for pure liquids or solids can at normal conditions be set to unity. In dilute solutions, with proper choice of reference states, activities may be exchanged for molalities or molarities.

\subsubsection{Gibbs Phase Rule}

Any pharmaceutical process, be it a manufacturing step in the large scale production of an API or the granulation of an API with excipients, must always proceed under strict control and lead to the formation of a well-defined product. Such tight process control should be based on a highlevel understanding of the heterogeneous equilibriums involved (equilibriums involving several condensed phases) from the mixing of the starting materials until the formation of the product. The standard tool for handling heterogeneous equilibrium, the Phase Rule, formulated by J. W. Gibbs in 1876 (Findlay and Campbell 1945; Ricci 1966) states that the number of degrees of freedom in a system equals the number of components plus two, minus the number of phases present at equilibrium:

$$
\mathrm{F}=\mathrm{C}+2-\mathrm{P}
$$

where $\mathrm{F}=$ the number of degrees of freedom, $\mathrm{C}=$ the number of components and $\mathrm{P}=$ the number of phases. The number 2 stands for the two variables of temperature and pressure. If any of these two is kept constant this number becomes 1; if both are constant it becomes 0 .

In terms of the Phase Rule a system is an isolated portion of matter subject to a limited range of specified variations. A component is the minimum number of chemical compounds needed to describe each phase in such a system. This number may be found by counting all independent chemical compounds and then, subtracting all chemical reactions and other restrictive conditions (material balance, charge balance and so forth) occurring in the system. Finding the correct number and identity of the components in a system sometimes requires some consideration (Alper 1999) but for the systems described in this text it will be straightforward.

A phase is an internally homogeneous part of the system, which is physically separated from other parts of the system by a surface (called the phase boundary), which makes it mechanically separable from all other phases in the system. Even though a single phase is subdivided into many smaller subparts, like oil droplets in water or crystals in a saturated solution, all the small parts will still constitute one phase, as long as the composition in each of the tiny fragments is the same. It should, however, be noted that different crystals of a solid solution, even though they altogether constitute one single phase, do not always have exactly the same chemical composition.

In this text only temperature, pressure and composition will be used as variables. The number of these, which can be varied without causing a change in another of the variables or the appearance or disappearance of a phase, is called the number of degrees of freedom or the variance. The term composition can be used to describe the amounts of each component in a phase, but it may also be used to describe the amounts of each component in the whole system. To clarify this the terms phase composition and total composition will be used. 


\subsubsection{One-Component (Unary) Systems}

The relations between the phases of a single substance can be plotted in a unary phase diagram as in Figure 1.7.

It is important to note that all phase transformations between the various phases in a phase diagram proceed at thermodynamic equilibrium, that is

$$
\text { Phase } \mathrm{A} \leftrightarrows \text { Phase } \mathrm{B}
$$

Such equilibrium is reversible and will proceed equally well in any of the two directions. If reversibility between two phases can be demonstrated then the two phases are very likely to be thermodynamically stable. When metastable equilibrium (see section 1.7) is discussed it is usually indicated with dotted lines in the phase diagram.

For a one-component system the Phase Rule states that $\mathrm{F}=3-\mathrm{P}$ so the number of phases at equilibrium can be one, two or three. In Figure 1.7 there are three one-phase areas, one for each of solid, liquid and vapour phase. In each of these there are two degrees of freedom, so that $\mathrm{T}$ and $\mathrm{p}$ can vary independently, without causing any phase changes. Between these areas there are three two-phase lines, along which $\mathrm{T}$ and $\mathrm{p}$ are interdependent, so that if one of them changes, the other will have to follow in order to stay on the line. If $\mathrm{T}$ and $\mathrm{p}$ change, so as to move away from the line into one of the one-phase areas, one of the two phases will have to disappear. Only in one point of the diagram is three phases occurring together in a state of equilibrium and this is the triple point marked tp in Figure 1.7. In this point $\mathrm{T}$ and $\mathrm{p}$ are strictly defined and cannot vary without one or two phases disappearing.

In Figure 1.7 a dotted line is drawn at $\mathrm{p}=$ atmospheric pressure. The line cuts the solid-liquid line at the 'normal' melting point and the liquid - vapour line at the 'normal' boiling point. It is important to note that the pressure axis in the figure symbolizes the vapour pressure of the substance and not the total atmospheric pressure. If the substance is water, the equilibrium vapour pressure over ice and water in equilibrium at $0.01^{\circ} \mathrm{C}$ will be $608 \mathrm{~Pa}$ (the triple point of water). In a sufficiently small and confined space this is the pressure that would be observed. Raising the temperature will melt the ice, but liquid and gaseous water will still be in equilibrium so the vapour pressure will increase along the liquid-vapour curve. When $\mathrm{T}$ reaches $100^{\circ} \mathrm{C}$ the vapour pressure is the same as the atmospheric pressure. If more heat is added to the system, two-phase equilibrium can only be maintained if $p$ increases. Since the atmospheric pressure in this case will function as a limiting piston, which will not allow an increase in p, water must, on further heating, transform from liquid to vapour. This is called the 'normal' boiling point.

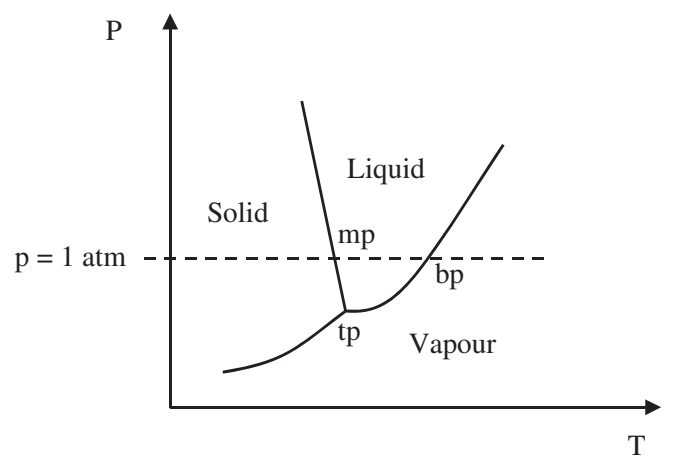

Figure 1.7 A part of a unary phase diagram 
It should be noted that along the two-phase lines the chemical potentials of the two co-existent phases are the same, so that $\mu_{\mathrm{i}}$ (phase 1$)=\mu_{\mathrm{i}}$ (phase 2). Similarly, the chemical potentials of the three phases in the triple point are also equal.

Proceeding past a two-phase line in a unary phase diagram means that one phase is transformed into another, so that a phase transition occurs. The slope of the two-phase line close to this phase transition can be obtained from the Clausius - Clapayron law

$$
\mathrm{dp} / \mathrm{dT}=\Delta \mathrm{S} / \Delta \mathrm{V}=\Delta \mathrm{H} /(\Delta \mathrm{V} \times \mathrm{T}),
$$

where all variables are measured at the phase transition point. It should be noted that a phase transition between solid and liquid can occur at any point along the solid-liquid two-phase line but if one of the two variables ( $\mathrm{p}$ or $\mathrm{T}$ ) is constant, then the phase transition can only occur at one point, for example the 'normal' melting point.

\subsubsection{Two-Component (Binary) Systems}

The phase diagram of a binary system of two pharmaceutical solids A and B, which do not form any mixed compounds, is shown in Figure 1.8. Along the two abscissa-axes are indicated the percentage $\mathrm{A}$ and $\mathrm{B}$ (in weight or molar percentage) and along the ordinate the temperature. The diagram is a cross-section at $\mathrm{p}=1$ atmosphere which means that $\mathrm{F}=3-\mathrm{P}$ so the maximum number of phases at equilibrium is 3 .

The melting points of the pure, crystalline substances are indicated as $M_{p}(A)$ and $M_{p}(B)$. The top area of the diagram is one single-phase area where $\mathrm{A}+\mathrm{B}$ are melted to a clear miscible liquid. The separation between this area and the two-phase area $\mathrm{A}+$ liquid is the line of final melting (or primary crystallization). Along this line the melting point of pure A gradually decreases as A is mixed with increasing amounts of $\mathrm{B}$. A similar reasoning applies for area $\mathrm{B}+$ liquid.

Below the final melting line is the two-phase area $\mathrm{A}+$ liquid where crystals of pure $\mathrm{A}$ are in equilibrium with a melt. The phase-composition of the melt at a temperature $T_{a}$ is determined by drawing a line $\mathrm{x}-\mathrm{z}$, parallel with the abscissa, from the left ordinate, terminating at the final melting line. This line is called a 'tie-line' and its two end points indicate the 'phase compositions' of the two phases in equilibrium (pure A and melt with ca. $60 \% \mathrm{~A}+40 \% \mathrm{~B}$ ). To calculate the amounts of crystalline A and melt at a certain total composition the 'lever rule' is used. In the figure this is done for point y (total composition 30\% A + 70\% B). The $\%$-amount of pure A equals 100 times the distance $\mathrm{y}-\mathrm{z}$ divided by the distance $\mathrm{x}-\mathrm{z}$. The $\%$-amount of melt equals to $100 *(\mathrm{x}-\mathrm{y}) /(\mathrm{x}-\mathrm{z})$. Area $\mathrm{B}+$ liquid is treated similarly.

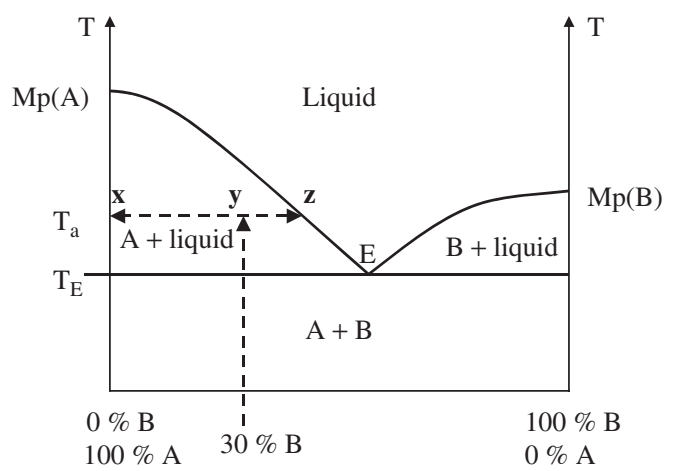

Figure 1.8 A two-component system with a eutectic melting point 
The two final melting lines meet at the eutectic point, E. In this invariant point the melt is in equilibrium with crystals of $\mathrm{A}$ and $\mathrm{B}$. $\mathrm{T}_{\mathrm{E}}$ is the lowest temperature at which a melt of $\mathrm{A}+\mathrm{B}$ can exist. Raising the temperature of any mixture of crystals of $\mathrm{A}+\mathrm{B}$, melting will always start at $\mathrm{T}_{\mathrm{E}}$ and continue until the final melting line is reached. DSC-scans of mixtures of $\mathrm{A}+\mathrm{B}$ with different total compositions will therefore all exhibit a first peak at $\mathrm{T}_{\mathrm{E}}$ followed by a second peak when the final melting line is reached. The peak temperature of the second peak will depend on the total composition.

Below the line through E, connecting the two ordinate axes is a two-phase area in which crystals of pure A and B exist in equilibrium. This area is a 'miscibility gap', indicating that the crystals of A and B are completely immiscible. If some B could fit, randomly into positions in the lattice of A a solid solution of B in A would exist. Solid solutions can be identified using a combination of DSC, chemical analysis and XRPD (Chien et al. 2005).

It should be noted that if component $\mathrm{A}$ in the binary phase diagram in Figure 1.8 was a pharmaceutical substance and B was a solvent, such as water, the general appearance of the phase diagram would be the same. However, the one-phase area would be a warm, under-saturated solution and the separating lines would be curves of maximum solubility (the solubility curves) and in areas $\mathrm{A}+\mathrm{B}$ and $\mathrm{B}+$ liquid, $\mathrm{B}$ would be ice and $\mathrm{M}_{\mathrm{p}}(\mathrm{B})$ would be $0{ }^{\circ} \mathrm{C}$.

\subsubsection{Three-Component (Ternary) Systems}

The only type of ternary phase diagram considered in this text is that with a pharmaceutical solid $\mathrm{A}$ and two solvents $\mathrm{B}$ and $\mathrm{C}$ as components. The simplest type of such a diagram is shown in Figure 1.9.

This diagram is drawn at constant atmospheric $\mathrm{p}$ and at constant $\mathrm{T}$. To read the total-composition in a point in the diagram, three lines through the point are drawn, each line parallel with one axis (see the three dotted lines in the figure). On any of the two points where such a line cuts two of the axes, the amount of the component is read (for example, $15 \% \mathrm{C}$ on any of the axes $\mathrm{A}-\mathrm{C}$ and $\mathrm{B}-\mathrm{C}$ in the figure). The solubility of $\mathrm{A}$ in solvents $\mathrm{B}$ and $\mathrm{C}$ at the temperature $\mathrm{T}$ are indicated as points ' $\mathrm{s}(\mathrm{A})$ in $\mathrm{B}$ ' and ' $\mathrm{s}(\mathrm{A})$ in $\mathrm{C}$ '. The solid line between these points is the line of maximum solubility (the solubility curve) for mixtures of A, B and C. It separates the one-phase area with under-saturated solution (area 'solution') from the two-phase area with solid A in equilibrium with a saturated solution (area 'A + solution'). To read the phase composition of the saturated solution at a point in this area, a tie-line from corner A is drawn through the point and ending

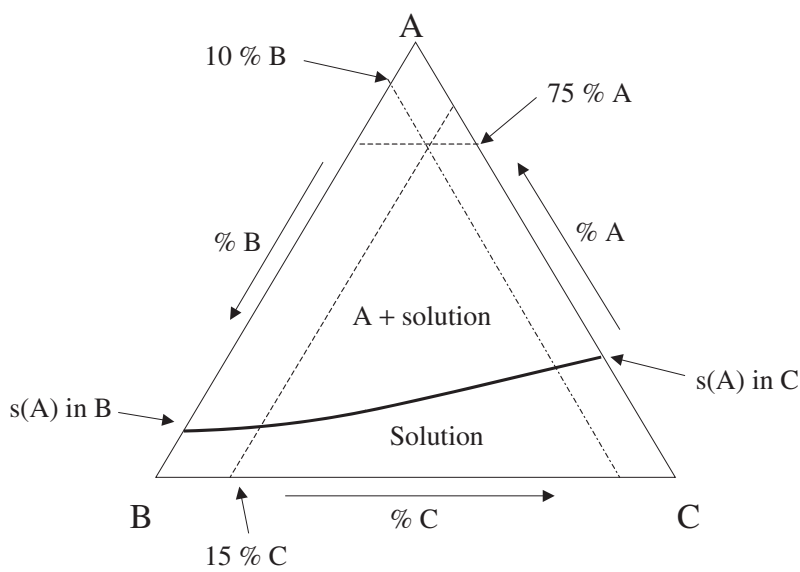

Figure 1.9 A ternary phase diagram of pharmaceutical solid $A$ and solvents $B$ and $C$ 
on the solubility curve. The solubility curve for this type of ternary phase diagram at $\mathrm{T}$ can be determined by slurrying solid A in differing amounts of B $+\mathrm{C}$ for a sufficiently long time. Samples of the saturated solution and of the solid phase are then withdrawn for analysis.

\subsection{Neutral Pharmaceutical Molecules in the Solid State}

As described in section 1.2, a pharmaceutical molecule may contain a lot of functionalities. All of these will be involved when molecules attract each other, form bonds and pack so as to form a crystal structure. The driving force for crystallization is to minimize the Gibbs energy of the crystals and to optimize several, competing, energy-minimizing, demands. The three main ones are (a) minimal energy of molecular conformations, (b) minimization of charge imbalances and (c) maximum packing density. These can be combined in a large number of ways but in only one will these be optimized and the energy minimized. That crystal structure represents the thermodynamically stable one under the conditions studied. Other combinations with energy levels close to this will be potentially metastable polymorphs (see Chapter 12).

The conformation of a pharmaceutical molecule in the most stable crystal structure will not necessarily be the same as that in the saturated solution from which crystallization progresses. The reason for this is that in solution the conformation is determined by interactions with itself (intramolecular bonding), with a large number of solvent molecules (even in highly concentrated solutions the number of solvent molecules is in high excess to the number of solute molecules) and sometimes also with a few other pharmaceutical molecules, whereas when packed in the crystal structure it must minimize its conformation in an environment consisting only of other molecules of its own kind. For instance, the atoms participating in an intramolecular hydrogen bond in the solution conformation may be much better used forming intermolecular hydrogen bonds in the crystal structure conformation.

When pharmaceutical molecules pack, the object is to minimize empty space. If no other types of intermolecular bonds are formed, the molecules are packed in a non-directional way, at van der Waals distances. The efficiency of this depends on the molecular extension in space. Many organic molecules can, with the aid of conformational flexibility, pack almost as efficient as spheres do. Closed packed spheres have a packing coefficient of 0.74 , whereas well-packed organic molecules may have values above 0.7 . From this it is concluded that when pharmaceutical molecules with very little unsaturated charges (by which is referred to atoms carrying lone electron pairs and protons with large electron deficits) form crystal structures, the two main driving forces are conformation minimization in combination with packing density maximization.

For molecules that contain unsaturated charges this simple picture changes. The unsaturated parts of the molecules will attract each other so as to form various types of hydrogen bonds, today a less simple phenomenon than 20 years ago (Jeffrey 1997; Desiraju and Steiner 1999; Steiner 2002). In contrast to dispersion bonds (London forces/van der Waals bonds) these bonds are directional, which means that they are less efficient from a packing point of view. Crystal structures of molecules forming extensive networks of hydrogen bonds thus have lower density than similar size molecules, which do not form hydrogen bonds. Packing coefficients of this type of crystal structures are usually in the range $0.65-0.70$.

This means that the third energy-minimizing demand, the minimization of charge imbalances, which is more-or-less synonymous with the formation of hydrogen bonds, to some extent counteracts the demand for maximized packing density. In fact there is much indication that saturation of unsaturated charges by means of hydrogen bonding is a primary demand in crystal structure construction, so that conformation and packing comes second. This is not so surprising considering the fact that a medium strong hydrogen bond is $10-100$ times stronger than a van der Waals bond (ca $0.2 \mathrm{kcal} / \mathrm{mole}$ ). 
As noted above, the concept of hydrogen bonds has lately been extended and today involves not only $\mathrm{X}-\mathrm{H}$...Y bonds, where $\mathrm{X}$ and $\mathrm{Y}$ are $\mathrm{N}, \mathrm{O}, \mathrm{S}$ and halogens but also hydrogen bonds where $\mathrm{Y}$ is different types of $\pi$-systems and where $\mathrm{X}$ is a carbon atom bonded to an electronrich atom (Desiraju 1995; Hunter et al. 2001). All of these types may thus be involved in the formation of the most stable crystal structure of neutral pharmaceutical molecules. However, as the strongest of these will dominate, traditional hydrogen bonds are still most important. In fact there is good evidence that in most cases all 'good' hydrogen bond donors and acceptors are used in the formation of organic molecule crystal structures (Etter 1990). It seems that classical hydrogen bond donors are active in hydrogen bonding in almost all cases.

Crystal structures of pharmaceutical molecules are determined from single crystal X-ray diffraction data collected from crystals of approximate size between $0.1-0.5 \mathrm{~mm}$. The data obtained from such investigations include unit cell parameters and positional and thermal parameters for all atoms. From these, pictures of single molecules, showing conformation, and pictures of the packing and intermolecular bonding can be drawn. In addition information about distances and angles between atoms, theoretical density, void volume and packing coefficient, thermal motion of the atoms, absolute configuration around chiral atoms, and so forth, are calculated. The analysis of the crystal structure of a neutral pharmaceutical molecule involves several steps such as

- identifying areas with weaker or stronger bonding;

- identifying hydrophobic and hydrophilic areas;

- identifying whether intermolecular hydrogen bonds are isolated or if they form chains, sheets or 3-dimensional networks;

- identifying $\pi-\pi$-stacking and other $\pi$-interactions;

- identifying areas with large thermal parameters, showing the presence of static (atoms, groups or molecules in incorrect positions) or dynamic disorder (atoms, groups or molecules which move due to thermal motion).

Taken together, this data presents a lot of information, which can be used to understand the behaviour of the molecule in the solid state. Please refer to more elaborate information in Chapter 2 about X-ray diffraction.

\subsection{Salt Formation and Acid-Base Equilibrium}

When biopharmaceutical or solid-state properties limit the use of the acid or base form of a drug substance, the way forward is the preparation of salts. These will have different properties, the most important ones being enhanced aqueous solubility and dissolution rate.

If the drug substance is an acid, mixing a solution of $n$ moles of the substance, with a solution of $n$ moles of a sufficiently strong base, and then crystallizing it will prepare a 1:1 salt. If the drug substance is a base, the procedure is similar using a sufficiently strong acid. For polyfunctional acids or bases it is also possible to prepare salts with other molar ratios.

For a Brønstedt acid HA in water, the protolytic constant $\mathrm{K}_{\mathrm{A}}$ is defined by the equilibrium

$$
\mathrm{HA} \leftrightarrows \mathrm{H}^{+}+\mathrm{A}^{-}, \mathrm{K}_{\mathrm{A}}=\left[\mathrm{H}^{+}\right]\left[\mathrm{A}^{-}\right] /[\mathrm{HA}] \text { and } \mathrm{pK}_{\mathrm{A}}=-\lg \mathrm{K}_{\mathrm{A}}
$$

where the square brackets symbolize molar concentrations. The stronger the acid the lower is the $\mathrm{pK}_{\mathrm{A}}$ value (very strong acids have values below zero). Every acid has a corresponding base, with which it forms an acid-base pair, for example $\mathrm{A}^{-} / \mathrm{HA}$ above. The corresponding base of a strong acid is a weak base and the corresponding base of a weak acid is a strong base. For a base B the corresponding relation is

$$
\mathrm{HB}^{+} \leftrightarrows \mathrm{H}^{+}+\mathrm{B}, \mathrm{K}_{\mathrm{A}}=\left[\mathrm{H}^{+}\right][\mathrm{B}] /\left[\mathrm{HB}^{+}\right] \text {and } \mathrm{pK}_{\mathrm{A}}=-\lg \mathrm{K}_{\mathrm{A}}
$$

Bases have high $\mathrm{pK}_{\mathrm{A}}$ values and every base has a corresponding acid. 


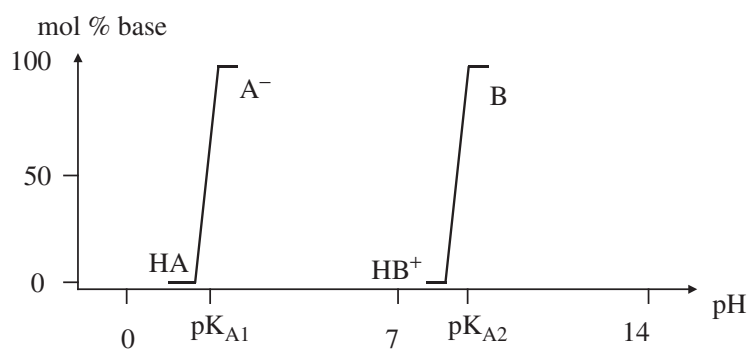

Figure 1.10 A diagram showing the relations of acid pairs $\left(A^{-} / H A\right)$ and base pairs $\left(B / H B^{+}\right)$

The relation between an acid HA and base $\mathrm{B}$ is schematically shown in Figure 1.10, where two 'titration' curves are drawn, one for each acid-base pair $\left(\mathrm{A}^{-} / \mathrm{HA}, \mathrm{pK}_{\mathrm{A} 1}\right.$ ca 2$)$ and $\left(\mathrm{B} / \mathrm{HB}^{+}, \mathrm{pK}_{\mathrm{A} 2}\right.$ ca 9). The curves symbolize the transformation from acid to base as the $\mathrm{pH}$ increases from just below the respective $\mathrm{pK}_{\mathrm{A}}$ to just above it. This is exactly what happens when an acid is titrated with a base in order to determine $\mathrm{pK}_{\mathrm{A}}$ for the acid. For a strong acid the titration curve is steep but for weaker acids the slope is less inclined. The same holds for strong and weak bases.

In order to obtain a salt an acid $\mathrm{HA}$ and a base $\mathrm{B}$ are mixed, which react to form $\mathrm{A}^{-}$and $\mathrm{HB}^{+}$ according to

$$
\mathrm{HA}+\mathrm{B} \leftrightarrows \mathrm{A}^{-}+\mathrm{HB}^{+}, \quad \mathrm{K}=\left[\mathrm{A}^{-}\right]\left[\mathrm{HB}^{+}\right] /[\mathrm{HA}][\mathrm{B}] \text { and } \mathrm{pK}=-\lg \mathrm{K}
$$

For enhancement of products $\mathrm{A}^{-}$and $\mathrm{HB}^{+}$this equilibrium must be strongly displaced to the right, consequently $\mathrm{K}$ must be at least in the order of $100-1000$. Since the salt equilibrium is the sum of

$$
\begin{array}{ll}
\mathrm{HA} \leftrightarrows \mathrm{H}^{+}+\mathrm{A}^{-}, & \mathrm{K}_{\mathrm{A} 1}, \mathrm{pK}_{\mathrm{A} 1} \\
\mathrm{H}^{+}+\mathrm{B} \leftrightarrows \mathrm{HB}^{+}, & \mathrm{K}_{\mathrm{A} 2},-\mathrm{pK}_{\mathrm{A} 2} \\
\hline \mathrm{HA}+\mathrm{B} \leftrightarrows \mathrm{A}^{-}+\mathrm{HB}^{+}, & \mathrm{K}=\mathrm{K}_{\mathrm{A} 1} / \mathrm{K}_{\mathrm{A} 2}, \mathrm{pK}=\mathrm{pK}_{\mathrm{A} 1}-\mathrm{pK}_{\mathrm{A} 2}
\end{array}
$$

then $\mathrm{K}=100-1000$ corresponds to $\mathrm{pK}=\mathrm{pK}_{\mathrm{A} 1}-\mathrm{pK}_{\mathrm{A} 2}$ of around 2 to 3 . Thus, the two curves in Figure 1.10 must be separated at least $2-3 \mathrm{pH}$-units for a salt to form.

Once the salt HBA has formed it will be stable as long as $\mathrm{pH}$ is somewhere between the two titration curves. One must keep this in mind when mixing the salt with solvents or excipients, so that these do not shift $\mathrm{pH}$ outside this interval. An especially troublesome substance in this case is water. Even though fresh de-ionized water will have a $\mathrm{pH}$ around 7 and no buffer capacity, if exposed to air, its properties will change rapidly due to the uptake of $\mathrm{CO}_{2}$ from surrounding air. This will cause $\mathrm{pH}$ to shift to an equilibrium value of approximately 5 and also to provide the water solution with a buffer capacity. Thus, mixing a salt with a stability interval of 1-4 (or 6-8) with water will transform the salt to the base (or acid).

Once acid and base have been mixed, an analysis for salt formation, by comparing with analyses of the starting materials, must be made. In solution this may be done using H-NMR or Raman spectroscopy. The analysis of the crystalline material may be done using XRPD, Raman or solidstate NMR. Usually this is quite straightforward but complications arise if either of the acid or base is noncrystalline or if polymorphism occurs.

Figure 1.11 shows a two-component phase diagram for a 1:1 salt (denoted as HBA in the figure) and its corresponding crystalline acid and base (denoted HA and B with melting points $\mathrm{M}_{\mathrm{p}}(\mathrm{HA})$ and $\mathrm{M}_{\mathrm{p}}(\mathrm{B})$ in the figure). The salt forms eutectics with both acid and base (eutectic melting temperatures are denoted $\mathrm{T}_{\mathrm{E} 1}$ and $\mathrm{T}_{\mathrm{E} 2}$ in the figure) and thus has a normal (congruent) melting point denoted as $\mathrm{M}_{\mathrm{p}}(\mathrm{HBA})$ in the figure (less common salts with incongruent melting points also 


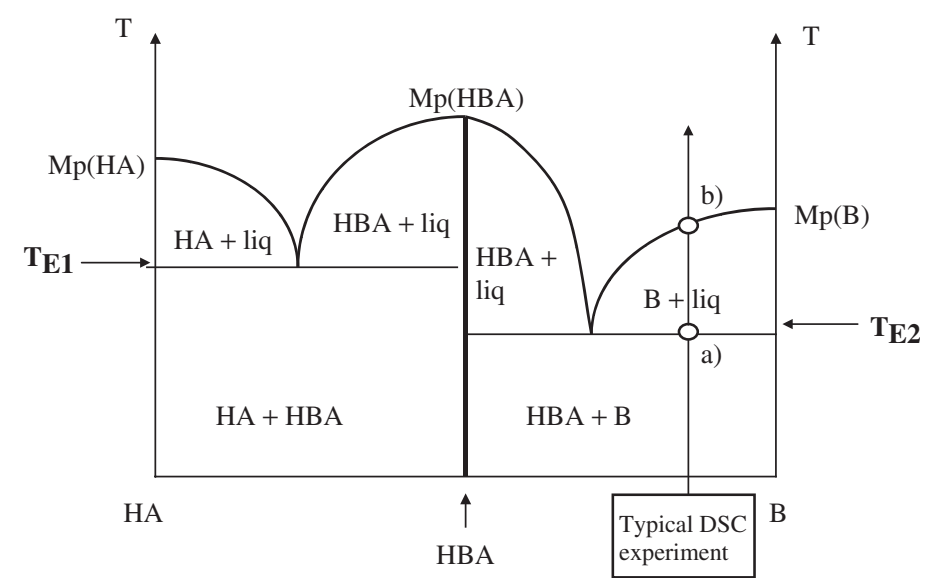

Figure 1.11 A typical phase diagram for a 1:1 salt and its corresponding, monofunctional crystalline acid and base - the diagram is drawn in molar \%

appear - Purdon and Slater 1946). There are also two miscibility gaps, denoted HA + HBA and $\mathrm{HBA}+\mathrm{B}$. The melting point of the salt is in this case higher than those of the acid and base, but it may just as well be lower. A phase diagram of the type in Figure 1.11 can be obtained using a DSC to study the melting behaviour of various mixtures of HA + HBA and B+HBA. In the right side of the figure the propagation of such a DSC-experiment is shown by an arrow with two circles. The circles show two melting events that will occur in the DSC curve: first the eutectic melting point (at the circle a) and then the final melting (at circle b). From such an experiment the circled data (a) and (b) are plotted into the figure and after 5-10 such DSC experiments the whole diagram can be drawn. The presence of enantiotropic polymorphs makes the diagram slightly more complicated. If the acid or the base (or both) is polyfunctional, new salts may also appear in the molar positions 1:2, 2:1 and so forth but the system will still remain a two-component system.

The solubility of a salt as a function of $\mathrm{pH}$ is often measured in buffered systems. Obtained in this way, the solubilities are not correct because the buffer ions introduce more components into the system. Thus, determining the solubility of a chloride salt in a phosphoric acid buffer will not give the solubility of the chloride salt in water at different $\mathrm{pH}$ but rather the solubility of some potentially other species (for example, a hydrogen phosphate salt) somewhere on a solubility curve in a four-component system.

This might still work as an approximation, but if correct data are desired the solubility must be determined in the pure ternary phase diagram $\mathrm{HA}-\mathrm{B}-\mathrm{H}_{2} \mathrm{O}$. This is done, at constant $\mathrm{p}$ and $\mathrm{T}$ by mixing different amounts of $\mathrm{HA}$ and $\mathrm{B}$ with different amounts of carefully deionized water, which is kept free of $\mathrm{CO}_{2}$. The result is then a phase diagram of the type shown in Figure 1.12. The diagram has one single-phase area, that of an un-saturated solution, three two-phase areas (HA + sol, $\mathrm{B}+$ sol and $\mathrm{HBA}+$ sol) where crystalline phases are in equilibrium with saturated solutions of varying composition and two three phase area $\left(\mathrm{HA}+\mathrm{HBA}+\mathrm{E}_{1}\right.$ and $\left.\mathrm{B}+\mathrm{HBA}+\mathrm{E}_{2}\right)$ where a eutectic solution is in equilibrium with crystals of two solid phases. The diagram conveniently shows in which areas the salt is actually stable and in which it is not.

Acidic drug substances, such as carboxylic acids, will form salts with alkaline salt formers. The most frequently used ones are alkali or alkaline earth metal hydroxides or carbonates, so metal salts of acidic drug substances are quite common. Their properties are very much dominated by the metal ion, striving to bind a certain number of atoms around it, thus forming a coordination sphere. The coordination is achieved by ion - dipole bonds, which are stronger than the ordinary 


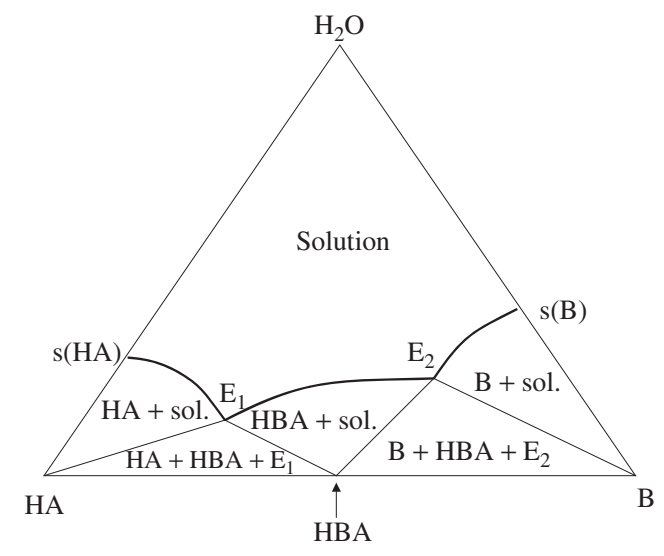

Figure 1.12 A ternary phase diagram of a mono-functional acid and base in water. E1 and E2 are eutectic points and $s(B)$ is the solubility of $B$ in water. The diagram is drawn in molar \%

bonds in molecular crystals. This is one of the reasons why metal salts often have higher melting points than the corresponding acidic drug substance, but on the other hand involve more solvate formation (see solvates and hydrates in section 1.6).

Since the coordination number in the solid state is primarily determined by the size of the metal ion and secondly by the charge, this varies as $\mathrm{K}^{+}>\mathrm{Ca}^{2+}>\mathrm{Na}^{+}>\mathrm{Mg}^{2+}$. Thus, whereas crystal structures with $\mathrm{K}^{+}$may contain 10 coordinated atoms per $\mathrm{K}^{+}$-ion, those of $\mathrm{Mg}^{2+}$ rarely exceed 6 (Poonia and Bajaj 1979). The fact that many drug molecules are quite large and often only contain one or two groups of interest to the metal ion means that metal salts will have a strong tendency to add small solvent molecules to complete its coordination shell (see section about solvates and hydrates). To further compensate for the lack of atoms, sharing of atoms between several metal ions often occur. Not so surprisingly, water oxygen atoms forming bridges between such polyhedra are common. Since the polyhedra may share corners, edges or faces, the resulting crystal structures may involve anything from isolated polyhedra to layers of polyhedra, with the drug molecules residing between the layers.

In addition to metal salts there are a number of organic alkaline salt formers, for example derivatives of the ammonium ion. The crystal structures of salts of these will have features similar to those of alkaline drug substances (typically various types of amines and amides) and acidic organic counter ions (typically various types of carboxylic and sulfonic acids) discussed below.

Since amines, amides, carboxylic and sulfonic groups contain protons bonded to oxygen and nitrogen, which are strong hydrogen bond donors and acceptors, the crystal structures of these types of salts are very much dominated by hydrogen bonding. In fact, the very strong ionic bonds between simple ions in inorganic salts, formed as a result of electrostatic attraction, are not present in drug salts. There are several reasons for this. The negative charges are more delocalized in the larger organic anions, and hydrogen atoms, which do not form simple ionic bonds, usually carry most of the positive charge. Thus, in these types of salts the charge carriers are attracted towards each other, but the link between them is always one or several hydrogen bonds. The result is anything from the formation of a simple hydrogen bonded anion-cation dimer, to complex, threedimensional hydrogen bond systems. These types of crystal structures are less dependent on solvent molecules, so hydration and solvation is less common than for metal salts.

Various inorganic acids are also used as salt formers, the most common ones being hydrochloric and hydrobromic acid and the oxo-acids of sulphur and phosphorous. Since all of these anions, including $\mathrm{Cl}^{-}$and $\mathrm{Br}^{-}$, are also strong hydrogen bond acceptors, the crystal structures tend to 
also be strongly hydrogen bonded. As an example, when chloride salts are formed with drugs containing amines, the chloride ion almost always accepts a hydrogen bond directly from the protonated amine. In this way the electrostatic attraction is also maximized. Due to their smaller size, inorganic acids usually give salts with higher melting points than those of larger organic acids (Giron and Grant 2002).

\subsection{Polymorphs, Solvates and Mixed Crystals}

Polymorphism is defined in different ways by different authors but in general it is based on the possibility of a molecule to form significantly different crystal structures in which the molecules are identical with regard to atom types and covalent bonding sequence. As with many other definitions there are unfortunately 'grey zones' (Bernstein 2002) but, being rarely encountered, they will not be discussed here. It should be noted that in order to discuss polymorphs of a substance there must be at least two polymorphs identified.

A very important feature is that different polymorphs have different physical properties such as solubility, melting point, density, crystal habit and so forth. The magnitude of the differences are usually related to the magnitude of difference in the crystal structures so that when the crystal structures are very similar so are the physical properties, whereas very different crystal structures give rise to larger differences in the physical properties.

There are several ways to analytically distinguish between different polymorphs. The gold standard is to use high-resolution XRPD (or single crystal XRD if large enough crystals are available) but also spectroscopic and thermo-analytical methods can usually be applied. It is good practice to combine more than one analytical method.

Polymorphic systems are usually divided into two groups, enantiotropic and monotropic. Unfortunately the definition of enantiotropy is not entirely clear. Therefore, in order to simplify things, this text will only refer to ambient pressure polymorphism and there define an enantiotropic system as one involving several thermodynamically stable (enantiotropic) polymorphs. In a monotropic system, on the other hand, there is, at ordinary pressures, only one thermodynamically stable polymorph. In any of the two types of systems there may exist a number of unstable polymorphs, all of which are referred to as monotropic polymorphs. More complete, but not completely similar, descriptions of enatiotropy and monotropy, are given by Findlay and Campbell (1945) and Ricci (1966).

In an enantiotropic system each polymorph has a temperature interval, in which it is stable and outside of which it is unstable. This means that it is possible to rank the polymorphs from that stable in the lowest temperature interval up to that stable in the highest temperature interval. In the phase diagram in Figure 1.13 there is one low-temperature and one high-temperature polymorph, A and $\mathrm{B}$, respectively. The line from $\mathrm{x}_{1}$ to $\mathrm{x}_{2}$ is drawn at ambient pressure. Heating low-temperature polymorph $A$ from point $x_{1}$, nothing special will occur until $t_{A B}$, the phase transition point from polymorph A to B is reached. If this point is passed slowly enough to maintain thermodynamic conditions, all of A will transform to the high-temperature polymorph B, which on further heating will melt at point $\mathrm{m}_{\mathrm{B}}$. If, however $\mathrm{A}$ does not transform to $\mathrm{B}$ at point $\mathrm{t}_{\mathrm{AB}}$, or indeed anywhere between $t_{A B}$ and $m_{A}$, its meta-stable melting point, it will melt there. Since this melt will be meta-stable (and supercooled) with regard to polymorph B, it may or it may not crystallize to polymorph $\mathrm{B}$ anywhere between $\mathrm{m}_{\mathrm{A}}$ and $\mathrm{m}_{\mathrm{B}}$.

For simple molecules the crystallization process between points $\mathrm{m}_{\mathrm{A}}$ and $\mathrm{m}_{\mathrm{B}}$ and the phase transition $\mathrm{A}(\mathrm{s}) \leftrightarrows \mathrm{B}(\mathrm{s})$ at $\mathrm{T}=\mathrm{t}_{\mathrm{AB}}$ or between $\mathrm{t}_{\mathrm{AB}}$ and $\mathrm{m}_{\mathrm{A}}$, will usually be observed. However, for larger and more complex molecules, or in crystal structures that would require significant molecular re-arrangement for a phase transition, they become more and more superheated, so that the solid-to-solid phase transitions usually do not occur at all and crystallizations become 


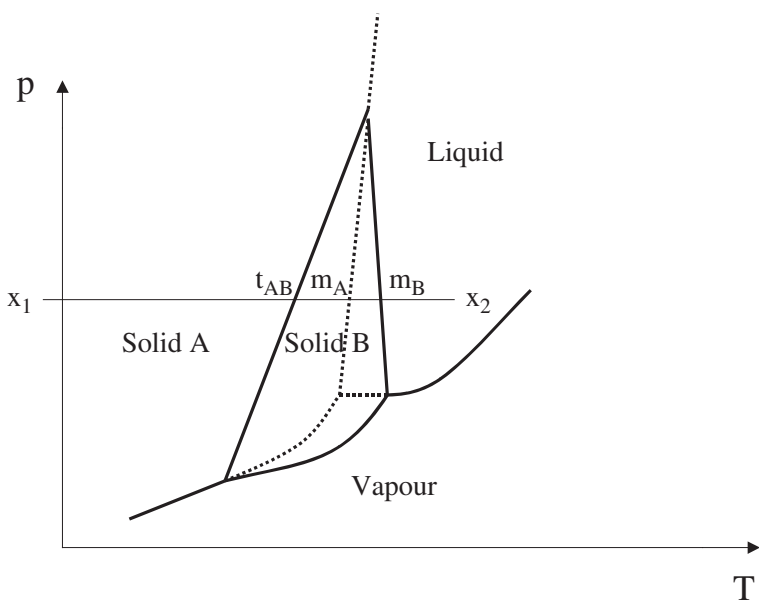

Figure 1.13 A unary phase diagram with two enantiotropically related polymorphs; solid lines indicate stable equilibrium and dotted lines metastability

very much heating-rate dependent. This must be kept in mind when evaluating DSC-traces of polymorphic substances. In order to obtain reliable data for solid-to-solid phase transformation temperatures competitive slurry experiments (see section 1.7) should be performed.

Theoretically a monotropic system has one thermodynamically stable polymorph from $0 \mathrm{~K}$ up to its melting point. In practice, investigations down to $0 \mathrm{~K}$ are not done, so a monotropic system is considered when one polymorph is thermodynamically stable from the lowest temperature investigated up to its melting point. All other polymorphs in a monotropic system will have melting points below the stable one.

To investigate if a system, containing several polymorphs, is monotropic or enantiotropic, one may perform competitive slurry experiments (see section 1.7) at the lowest possible temperature and compare these with the melting points of the polymorphs. The polymorph, which competes successfully at the lowest temperature investigated, is the stable low temperature polymorph. If this also has the highest melting point, the system is monotropic. If there exist polymorphs having higher melting points these are high-temperature polymorphs and the system is enantiotropic.

The different thermodynamic stability of polymorphs is an estimate of their different Gibbs free energies. These are reflected in the solubilities, which at a certain temperature, ranks polymorphs according to their stability. It should be noted that the same ranking is obtained in any solvent. At solid-to-solid phase transition points, for two enantiotropic polymorphs, the solubilities and the Gibbs free energies of both polymorphs are the same. Thus, solubility curves as a function of temperature always cross at phase transition points. This is the basic idea behind competitive slurry experiments (section 1.7).

Crystal structures of different polymorphs show that polymorphism occurs as a result of differences in molecular conformations, hydrogen bonding and molecular packing. For rigid molecules, polymorphism may often be a result only of packing differences. However, for flexible molecules, because differences in conformations inevitably result in different packing, polymorphism is usually the result of the combination of the above factors.

The gradual heating of a polymorph results in an increase in thermal motion leading to a decrease in density. At a phase transition point a more radical change in the crystal structure occurs. This may be a conformational change, a molecular rotation or a molecular translation, or the combination of these. 
A famous enantiotropic system is that of ammonium nitrate (Dellien 1981), which has five enantiotropically related polymorphs ranking in the order $\mathrm{V}$ (from low temperature to $-17^{\circ} \mathrm{C}$ ), IV $\left(-17-32^{\circ} \mathrm{C}\right)$, III $\left(32-84^{\circ} \mathrm{C}\right)$, II $\left(84-125^{\circ} \mathrm{C}\right)$ and I $\left(125\right.$ to melting point at $\left.170^{\circ} \mathrm{C}\right)$. In polymorph $\mathrm{V}$ all ammonium hydrogen atoms and all nitrate oxygen atoms are involved in hydrogen bonding. On heating, thermal motion gradually weakens the hydrogen bonds, and at each phase transition point, hydrogen bonds are gradually broken/rearranged. In the high-temperature polymorph I, no hydrogen bonds at all exist. As a result the ammonium and nitrate groups rotate freely and thus pack as spheres (Choi and Prask 1982, 1983).

It is a well established fact that crystallization from different solvents sometimes consistently yields different polymorphs. This is a kinetic phenomenon which, although not entirely understood, probably depends on that (a) a pharmaceutical molecule may take different conformations in different solvents; (b) different solvents provide different activation energies for primary nucleation (section 1.7) and (c) different solubilities leads to different degrees of solvent - solute and solute - solute interactions (Bernstein et al. 1999).

A big problem with polymorphism is that it is not possible to prove that a certain polymorph, under certain conditions, is the most stable one. Having found several polymorphs, the testing of these at the conditions desired, can only show that one is more stable than the others. Thus more stable polymorphs might appear, and if the new polymorph is so dominant that the old one disappears and never can be made again, the situation becomes problematic (Dunitz and Bernstein 1995). Since a disappearing polymorph is monotropic, the best way to avoid the risk that the polymorph chosen for pharmaceutical use is a disappearing one is to choose a polymorph, which seems to be thermodynamically stable.

Since this is indicated by the presence of reversibility, this is an important property to investigate. Having obtained a diagram of the type shown in Figure 1.14, will give good faith that all crystal modifications involved are thermodynamically stable. Adding enantiotropy to this will obviously further strengthen the case.

\subsubsection{Mixed Crystals}

The risk or possibility to observe different physical properties for a pharmaceutical substance because of polymorphism was discussed above. Another way to modify physical properties is to crystallize the pharmaceutical molecule in crystal structures with other types of molecules. This may be done deliberately or not and may result in stoichiometric or nonstoichiometric phases being formed.

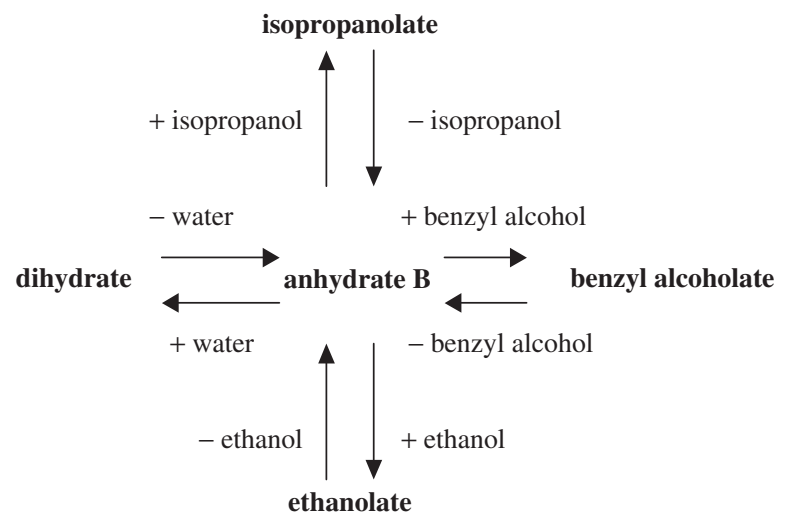

Figure 1.14 Reversibility between an anhydrate and a number of solvates 
The most obvious stoichiometric type of compounds is the salts, already discussed in section 1.4. Another type is co-crystals, which are formed by mixing two or more 'synthon forming' (see section 1.4) molecules in even molar ratios like 1:1, 2:1, 1:2 (the ratio is determined by the number of $\mathrm{H}$-bond donors on one molecule and acceptors on the other) and so forth. In contrast to salts, the molecules in co-crystals have not exchanged protons, so no ions are present in the solid. Instead co-crystals involve various types of weak to strong hydrogen bonding and/or $\pi$ bonding, by means of which, crystal structures with anything from isolated molecular assemblies up to three-dimensional networks are formed. Co-crystals may be obtained by mixing solutions of the components and crystallizing (Morissette et al. 2004), or by extensive grinding of the solid ingredients (Trask and Jones 2005). Co-crystals with molar ratio 1:1 have similar phase diagrams as those discussed for salts (Figures 1.11, 1.12).

A third way to make mixed crystals is to use one molecule to build a crystal structure (the host) and to let other molecules (guests) take positions in the structure, in which they are isolated from each other. Such a compound is usually referred to as a host-guest compound. These may be stoichiometric or non-stoichiometric and they include a number of subtypes such as clathrates, intercalation compounds and zeolites (Müller et al. 1995).

A less deliberate occurrence of mixed crystals is the formation of solid solutions (see also section 1.3). This phenomenon appears when molecules in a certain lattice position in the crystal structure of a compound are exchanged, in a random way, for similar but foreign molecules. Solid solutions are common in metals and inorganic salts (Ricci, 1966), where substitutions may occur all the way between $0-100 \%$. Such large substitution ranges may also occur in molecular crystals if the molecules are structurally similar (Albertsson et al. 1978). If small amounts of impurities cannot be removed by means of crystallization solid solutions should be suspected. It should be noted that the formation of a solid solution, from a pure compound, does not involve a phase change.

\subsubsection{Solvates and Hydrates}

When pharmaceutical and solvent molecules form mixed crystals they are called solvates, with the subgroup hydrates when the solvent is water. Solvates may be thermodynamically stable or not, with the stable ones being much more common.

The thermodynamically unstable solvates form for kinetic reasons, often as a result of an unstable anhydrate adding solvent molecules to its crystal structure, in order to stabilize it. Typical ways to prepare unstable solvates are thus to expose a metastable ansolvate to solvent vapour or to slurry it quickly in a solvent. Unstable solvates may also appear as a result of fast evaporative crystallizations.

A thermodynamically stable solvate is only stable in contact with its solvent, and in a certain temperature interval. Thus, all solvates except hydrates are thermodynamically unstable in air even though they sometimes may be kinetically stabilized. For instance, a hydrate may persist in a metastable state for long times at low relative humidities and an anhydrate may persist in a metastable state for long times at high relative humidities.

Solvates usually exists in parallel with a thermodynamically stable ansolvate, so that at low solvent activity the ansolvate is more stable, whereas at high solvent activities the solvate is more stable. This means that, in air, a hydrate may be stable at high relative humidity, whereas its corresponding anhydrate is stable at low.

It is not clearly understood why stable solvates form but in some cases it is obvious that they form as a result of the pharmaceutical molecules not being able to pack sufficiently well, so small solvate molecules compensate for this by filling out voids. In other cases solvent molecules act as hydrogen bond acceptors or donors (or both) to 'unsaturated' hydrogen bonding positions on the drug molecule, thus creating significant lowering of the lattice energy. It is obvious that, because 
some compounds do not form solvates at all, some anhydrates are obviously stable enough to dominate even in the chemical environment of pure solvents.

Solvates may be treated by means of Gibbs phase rule (Purdon and Slater 1946). If a hydrate with a 'normal' (congruent) melting point exists, the phase diagram will be of the type shown in Figure 1.15. There is, however, also the possibility that the hydrate does not melt but instead, at a certain temperature, decomposes into an anhydrate and a saturated solution. For such a hydrate the phase diagram will be similar to that in Figure 1.16. The temperature where the decomposition occurs is called the incongruent melting point.

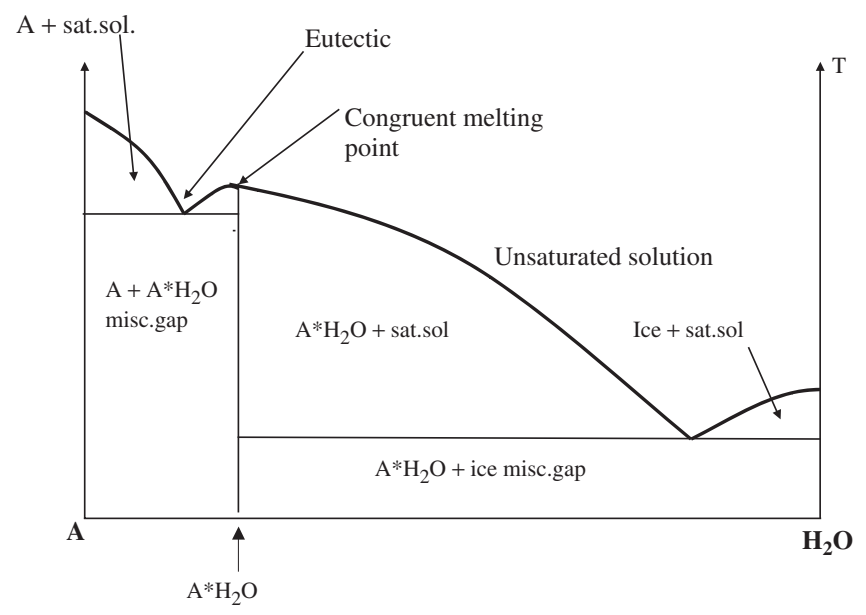

Figure 1.15 A binary phase diagram of pharmaceutical compound A and water, with a congruently melting hydrate

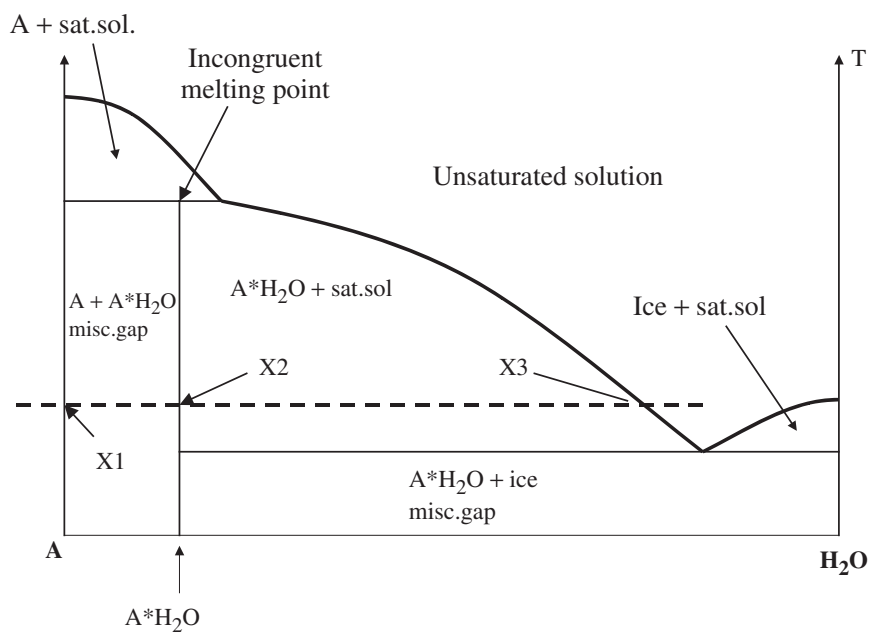

Figure 1.16 A two component system of pharmaceutical compound $A$ and water with an incongruently melting hydrate; the water vapour pressure variation along the line $x_{1}-x_{2}-x_{3}$ is shown in Figure 1.17 


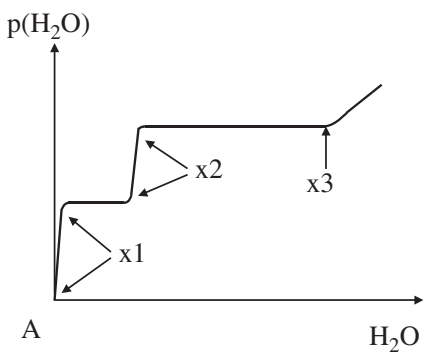

Figure 1.17 A two component system of pharmaceutical compound $A$ and water with an incongruently melting hydrate; the water vapour pressure variation along the line $x_{1}-x_{2}-x_{3}$ is shown in Figure 1.17

In aqueous systems, at ambient conditions, there will be water vapour present above the condensed phases. Along the isothermal line $\mathrm{x}_{1}-\mathrm{x}_{2}-\mathrm{x}_{3}$ in the binary system in Figure 1.16 the water vapour pressure will vary as shown in Figure 1.17. Starting at $\mathrm{x}_{1}$, the anhydrate has no water vapour pressure but as soon as some water is added the water vapour pressure will rise until some solid hydrate is formed. The vapour pressure must then be constant because in the two-phase area $\left(\mathrm{A}+\mathrm{A} * \mathrm{H}_{2} \mathrm{O}\right)$ there are no degrees of freedom (atmospheric pressure and $\mathrm{T}$ are constant so $\mathrm{F}=2-2+0$ ). When the one phase line of the hydrate is reached at $\mathrm{x}_{2}$ the last crystals of the anhydrate have disappeared and then the vapour pressure will rise until a new phase, the saturated solution has been formed and a new two phase area $\left(\mathrm{A}^{*} \mathrm{H}_{2} \mathrm{O}+\right.$ saturated solution) is entered. Continued addition of water will dissolve all hydrate and as the one phase area of nonsaturated solution is reached at $\mathrm{x}_{3}$, the vapour pressure will start to change again as a result of continued dilution.

It is quite important to note that the transformation from the stable anhydrate to the stable hydrate along the line $\mathrm{x}_{1}-\mathrm{x}_{2}$ will not just happen as an effect of water sorption. The crystal structures of an anhydrate and a hydrate are usually so different that they cannot transform into each other without dissolution and recrystallization. Most likely the anhydrate must be slurried in the two-phase area $\left(\mathrm{A}^{*} \mathrm{H}_{2} \mathrm{O}+\right.$ saturated solution) in order to be transformed.

Similarly, the hydrate usually cannot be transformed to the stable anhydrate just by drying. On the other hand there is no area in the binary phase diagram where the hydrate can be slurried, so as to transform it to the stable anhydrate. To find such an area one must add one more solvent and thus work with a three-component system instead. In Figure 1.18 the added component is ethanol. In the two-phase area $(\mathrm{A}+$ saturated solution) the stable hydrate may be slurry converted to the stable anhydrate.

Although not different from co-crystals or host-guest compounds, or even in some cases from solid solutions, hydrates and solvates have so far not been treated as such. Instead they have been classified with regard to analytical results as (a) isolated site solvates, (b) channel solvates and (c) metal ion associated hydrates (Morris 1999; Vippagunta et al. 2001).

According to the above authors isolated solvates give sharp DSC-peaks and weight losses in TGA, at temperatures close to the boiling point of the solvent. It is also stated that the solvent molecules take positions in the crystal lattice, in which they do not have any contact with each other. Since the boiling point of a solvent depends on intermolecular interactions between solvent molecules it is not clear if, or why there is a relation between the degassing temperature of isolated solvates and the boiling point of the corresponding solvent. Still, a high (or low) onset temperature of solvent loss, as observed with TGA or DSC, is usually an indication of a strong (or weak) interaction between the solvent molecules and the drug molecules.

Unfortunately, the definition of isolated solvates is not taken any further. For example, no specification is given with regard to whether the solvent molecules are passive guests in a host-guest type 


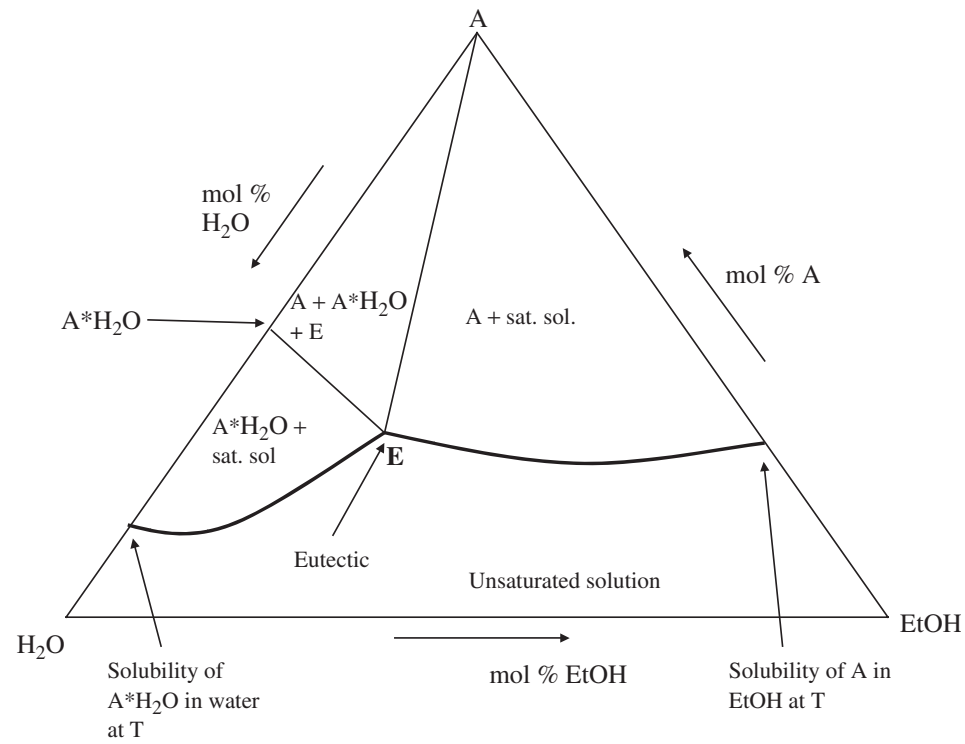

Figure 1.18 A ternary phase diagram for pharmaceutical substance $A$, water and ethanol, drawn at constant atmospheric pressure and constant temperature - the substance $A$ forms a monohydrate $\mathrm{A}^{*} \mathrm{H}_{2} \mathrm{O}$

of structure or whether they are active co-crystal formers. The possibility of both most definitely exists and could probably be used to give a more specific description of these types of solvates.

The channel hydrates are defined as those having solvent molecules, which are bonded to each other in channels or layers in the crystal structure. These give broad DSC peaks and less sharp weight losses in TGA, which often start at low temperature. Such hydrates can often be dehydrated without a phase transformation, so the XRPD patterns for the fully hydrated and dehydrated forms are similar, but show small but significant peak shifts. Typically, the water content in such hydrates varies with the relative humidity, so a gravimetric water vapour sorption experiment of such a hydrate would give an almost linear water uptake as a function of percentage relative humidity.

The third type of solvate involves metal ions and has already been touched upon in section 1.5. Since they contain solvate molecules bonded rather tightly to the metal ions, they show analytical behaviour similar to the isolated solvent type. However, it is quite common that the coordinated solvents are bonded differently and also that these solvates contain the isolated, uncoordinated type of solvent. Quite often dehydration using TGA thus proceeds in a stepwise manner. Between steps it may be possible to identify intermediate, crystalline phases.

Although not very common, there are also mixed solvates, where one of the solvents usually is water. Intermediate solvates may be obtained on desolvation.

It should be noted that many solvates, especially those that are not hydrates, lose their solvent very quickly when exposed to air. If filtered off and dried prior to analysis they are in many cases not observed. The only way to observe such solvates is to analyse them in a solvent slurry.

\subsection{Phase Transitions and Kinetics}

A solid crystalline phase of a pharmaceutical substance may be thermodynamically stable or unstable. A stable one has a certain interval of $\mathrm{p}$ and $\mathrm{T}$ where it is stable whereas the unstable 
Table 1.3 Factors affecting the formation of kinetic and thermodynamic phases

\begin{tabular}{lcc}
\hline Parameter & Thermodynamics & Kinetics \\
\hline State: & Wet & Dry \\
Time: & Long & Short \\
Temperature: & Constant & Varying \\
Composition: & Constant & Varying \\
Purity: & High & Low \\
Super-saturation: & Low & High \\
\hline
\end{tabular}

phase lacks such an interval. An unstable phase, which is encountered at any combination of $p$ and $\mathrm{T}$, or a stable phase, which is encountered outside of its stability-interval is in a metastable state. The reason for metastability is that there exists an activation energy, Ea, which stops the metastable phase from transforming to the stable phase. The presence of a metastable phase shows that kinetics instead of thermodynamics prevails. In order to avoid the metastable phase or to transform it to a thermodynamically stable phase one must work thermodynamically. Factors governing kinetic and thermodynamic phases are summarized in Table 1.3.

The time it will take for all molecules to transform from one phase to another depends on the magnitude of Ea. The fraction of molecules on a certain energy level is given by the Bolzmann factor $\mathrm{e}^{-\mathrm{Ea} / \mathrm{RT}}$. Thus, the higher Ea for a phase transition is, the fewer molecules will have energy enough to overcome it and the longer time it will take for all molecules to transform. However, $\mathrm{Ea}$ is not a constant. If a phase transition is driven too fast it will become super-cooled or superheated, which might increase Ea. An example of this is when evaporating a solution too quickly so that crowding appears. The crowding makes it harder for a molecule to take the necessary conformation and place in the crystal lattice, thus increasing the energy of activation. In some cases this will lead to the formation of a glass.

\subsubsection{Crystallization}

Crystallization may occur from a melt or from a solution. The driving force for the phase transition is the difference in Gibbs energy between the crystals and the saturated solution, $\mathrm{G}$ (sol)-G(cryst). This difference is zero when the solution is exactly saturated but as it becomes supersaturated G(sol) increases to $\mathrm{G}$ (sup). The supersaturation may be obtained by cooling, evaporation or addition of a solvent, in which the pharmaceutical substance has less solubility.

The event of crystallization from a supersaturated solution is considered to proceed in two steps: nucleation and crystal growth. Nucleation may be primary (homogeneous or heterogeneous) or secondary (already formed crystals break up to form new nuclei). Heterogeneous nucleation occurs on foreign surfaces (different types of equipment, solid impurities and so forth) and homogeneous nucleation occurs when no foreign surface exists on which nucleation can occur.

The theory of homogeneous nucleation (Jancic and Grootscholten 1984; Mullin 2001) describes the molecules as they move around in a homogeneous, supersaturated solution. They frequently collide and in some of these collisions intermolecular bonds form so that gradually larger and larger clusters are built up. However, these clusters are also subject to forces of decomposition. In order for crystallization to occur the clusters must reach a critical nuclei size. This is the minimum size needed, at prevalent conditions, to stabilize the particle from disintegration. In order to reach the critical size a certain energy input, which depends on the size of the particles surface compared to its total size, is needed. Since surface formation consumes energy, whereas bulk (interior) formation of a particle releases energy, the energy input to form a nucleus of the critical size is $\Delta \mathrm{G}($ critical $)=\Delta \mathrm{G}$ ( surface) $-\Delta \mathrm{G}$ (interior). The value of $\Delta \mathrm{G}$ (critical) works as an activation energy, so when this energy level is overcome crystallization may proceed (Figure 1.19). 


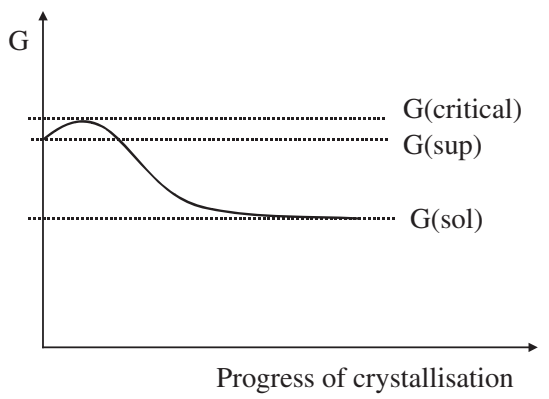

Figure 1.19 The variation of Gibbs free energy with the progress of crystallization

The rate of homogeneous nucleation may be expressed as $\mathrm{J}=\mathrm{Ae}^{-\Delta \mathrm{G} \text { (critical)/RT }}$. Theoretically the rate of homogeneous nucleation increases with increasing supersaturation and temperature but in practice, since the supersaturation affects the viscosity of the solution, it must not become too high. If so it might stop nucleation and subsequently lead to the formation of amorphous material.

In a phase diagram as Figure 1.8, when cooling a melt or solution from the one-phase liquid area, crystallization will not proceed with an observable rate until a certain level of supersaturation is reached. The area between the line from $\mathrm{M}_{\mathrm{p}}(\mathrm{A})$ to $\mathrm{E}$ and the area where nucleation occurs is called the metastable zone.

Since both heterogeneous and secondary nucleation lowers $\Delta \mathrm{G}$ (critical), they facilitate nucleation and make it possible to perform crystallization at lower relative supersaturation (the ratio between the actual concentration in the supersaturated solution and the concentration of the exactly saturated solution). Other ways to improve nucleation are to use varying types of energy input, such as stirring or ultrasonication (Mullin 2001; Meenan et al. 2002).

After successful nucleation the critical nuclei, which are believed to contain some 10-1000 molecules, will grow to macroscopic crystals. In this process molecules diffuse to the surface of the nuclei, where they are incorporated into the crystal structure. The size and number of crystals obtained are much dependent on supersaturation, so that high superaturation leads to many small crystals whereas low supersaturation leads to fewer and larger crystals. Size is also affected by Ostwald ripening, a process in which small crystals, due to their higher surface:volume ratio, have a higher energy and thus dissolve on the account of growth of the lower energy, larger crystals.

Crystal growth may also be severely affected by dissolved impurities (inhibitors). If these have groups, that can bind strongly enough to positions in the crystal structure they might block continued growth. In most cases this will only lead to modification of the crystal habit but in severe cases it can stop growth completely. If habit modification is actually desired, both the use of solvents as well as small amounts of specially chosen impurities (habit modifiers) may be used for this (Mullin 2001; Meenan et al. 2002).

The crystallization of polymorphs is often strongly affected by both nucleation and crystal growth conditions. If all of these are lumped into the term activation energy, $\mathrm{E}_{\mathrm{a}}$, the combined action of thermodynamics and kinetics can be described with regard to Figures $1.20-1.21$. If $E_{a}$ is much higher for the stable polymorph (solid line in Figure 1.20) it will not have a large chance of crystallizing even if the crystallization runs very slowly at low supersaturation. It would only appear if there were any means of lowering $E_{a}$, for example by changing the solvent. In this case the unstable polymorph will crystallize into a metastable state. If both polymorphs have similar levels of $E_{a}$, fast to moderate rates of crystallization will lead to the formation of both concomitantly, whereas very slow crystallization would preferentially give the stable polymorph. If, as in Figure 1.21, $\mathrm{E}_{\mathrm{a}}$ of the unstable polymorph was much higher than that of the stable polymorph, the unstable polymorph would never be observed. The phenomenon of a disappearing 


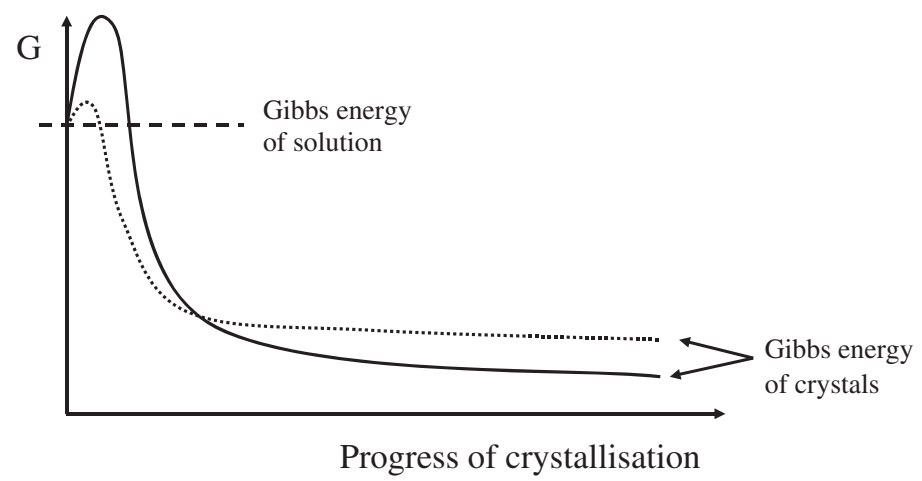

Figure 1.20 The progress of crystallisation for one thermodynamically stable and a thermodynamically unstable polymorph; in figure 1.20 (left side) the activation energy is much higher for the stable polymorph and in Figure 1.21 (right side) the activation energy is higher for the unstable polymorph

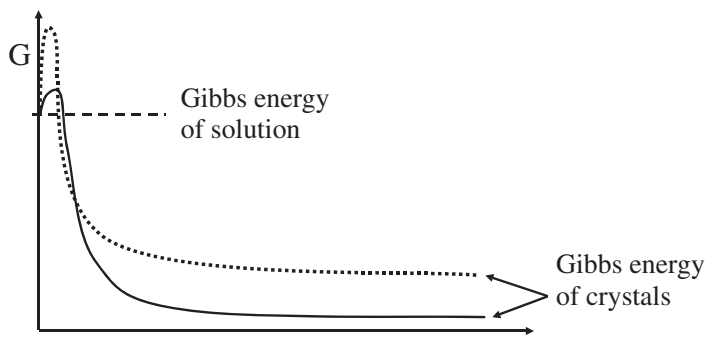

Progress of crystallisation

Figure 1.21 The progress of crystallisation for one thermodynamically stable and a thermodynamically unstable polymorph; in figure 1.20 (left side) the activation energy is much higher for the stable polymorph and in Figure 1.21 (right side) the activation energy is higher for the unstable polymorph

polymorph would at first have a diagram like that in Figure 1.20, but once the stable polymorph has nucleated its $\mathrm{E}_{\mathrm{a}}$ changes radically so that the diagram becomes like that in Figure 1.21.

\subsubsection{Solid-to-Solid Phase Transitions}

The process of transforming one solid crystalline phase to another means that one crystal structure is changed into another. This inevitably means that bonds are reallocated (broken, formed or both) and as a consequence parts of the molecules must move. The theoretical treatment of solid-tosolid phase transitions is quite similar to that of crystallization, including the two main events of nucleation and domain growth (Raghavan and Cohen 1975).

The translation of large pharmaceutical molecules in the solid state is not a simple task, so it is not surprising that, due to the extremely high activation energy (see Figure 1.22), pharmaceutical molecules seldom display solid-to-solid phase transformations. When they do occur, the transformations are often rather 'sluggish' and proceed over large temperature ranges. It should be noted that the high activation energy also is the reason why unstable polymorphs are observed as metastable.

With regard to this, transformations between polymorphs or between ansolvate and solvate should in practice always be performed as competitive slurry experiments. These experiments are 


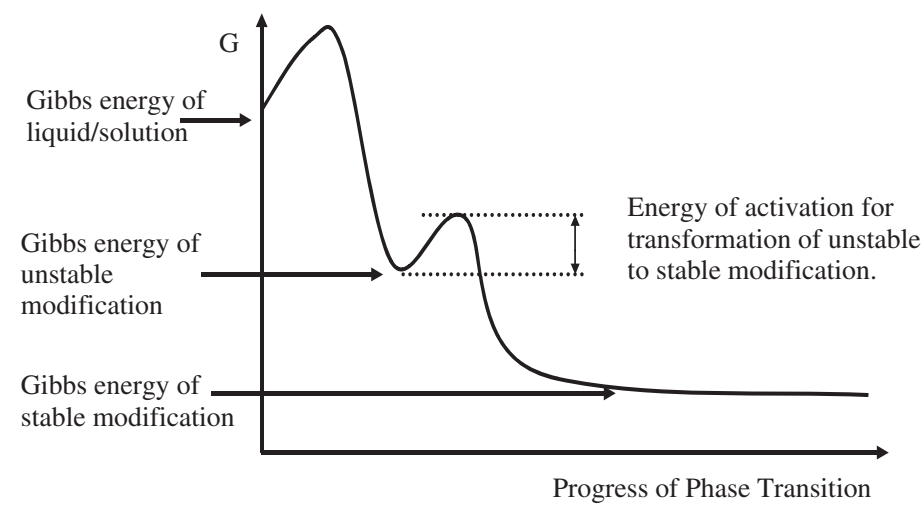

Figure 1.22 The progress of crystallization and a subsequent solid-to-solid phase transition

based on the fact that solubility curves as a function of temperature always cross at phase transition points. As two polymorphs with different solubilities (different thermodynamic stability) are mixed in a solvent, or in a mixture of solvents, the more soluble one will dissolve and the less soluble one will precipitate.

Competitive slurry experiments in combination with melting-point determinations, as described in section 1.6, can be used to determine if two polymorphs are related monotropically or enantiotropically. One exception appears when no melting points can be accessed due to chemical decomposition. In such a case, again, a competitive slurry experiment at the highest possible temperature might solve the problem. If it shows that one polymorph is stable at low temperature and the other at high temperature, this proves that the system is enantiotropic. If the same polymorph competes successfully at both temperatures no definite conclusion can be drawn and in such a case other methods must be applied, for example G -T-diagrams (Yu et al. 2000). If nothing else helps, the Buerger-Ramberger rules (Grant 1999; Bernstein 2002) can be applied. One must then remember that these are not rules but merely indications so they will not always provide a definite answer.

In order to determine the exact transition temperature for two enantiotropically related polymorphs, competitive slurry experiments will also work within the limitations noted above.

\subsection{Screening for 'Polymorphs' (Ansolvates and Solvates)}

In order to find as many anhydrates and solvates and polymorphs thereof, most pharmaceutical companies performs so called 'polymorph screening'. Such screens can be designed in slightly different ways but one should always make sure that all dimensional variables of importance are covered for the formation of different ansolvate and solvates and polymorphs thereof. For the formation of thermodynamically stable phases these variables are temperature, pressure and composition of API and solvent(s). This can be done by screening ternary phase diagrams as described in Figure 1.23. Ten different samples are prepared by mixing water and an organic solvent, in varying proportions in small vials, and then adding API until it is not dissolved, plus at least $5 \mathrm{mg}$ of extra solid in each vial. All vials are then capped and stirred isothermally for a period of 3-7 days. The saturated solution is then withdrawn (through a filter) from each vial and analysed for API (and solvents if necessary) and the 'wet' solid is analysed for identity with XRPD. From the data the isothermal section of the ternary diagram is plotted. This can be repeated at high (as close as possible to the solvent boiling point) and low (normally $3-5^{\circ} \mathrm{C}$ ) temperature 


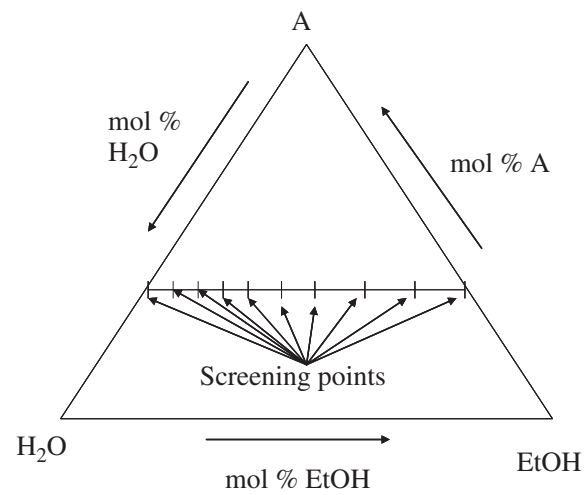

Figure 1.23 A description of how to design experiments to obtain an isothermal ternary phase diagram, with two solvent components (water and ethanol), and one solid component ( $A$ )

as desired. The procedure is then repeated for all organic solvents of interest. More information about this procedure can be described by Jarring et al. (2006) and Harris et al. (2008).

To capture metastable modifications one must also include the supersaturation variable into the screen.

\subsection{Summary}

Using a combination of crystallographic and thermodynamic knowledge together with good analytical instrumentation, most problems within the solid-state field of pharmaceutical substances can be understood and controlled. It is very important to know how to obtain thermodynamic data and also how to use these data in an optimal way (by using Gibbs Phase Rule). With this solid base of thermodynamic knowledge, kinetics becomes much easier to understand.

\subsection{Acknowledgements}

The author wishes to thank Professor Lars Kloo, at the Royal Institute of Technology in Stockholm for enlightening discussions.

\section{References}

Ahlneck, C., Sebhatu, T. and Angberg, M. (1994) Assessment of degree of disorder in crystalline solids by isothermal microcalorimetry. Int. J. Pharm. 104: 135-144.

Albertsson, J., Oskarsson, ^. and Svensson, C. (1978) X-ray study of budesonide: molecular structures and solid solutions of the (22S) and (22R) epimers of 11 $\beta, 21$-dihydroxi-16 $\alpha, 17 \alpha-$ propylmethylenedioxy-1,4-pregnadiene-3,20-dione. Acta Cryst. B34: 3027-3036.

Alper, J. S. (1999) The Gibbs Phase Rule revisited: interrelationship between components and phases. J. Chem. Educ. 76: 1567-1569.

Barcza, L. and Buvári-Barcza, A. (2003) Acid-base titrations in nonaqueous solvents and solvent mixtures. J. Chem. Educ. 80: 822-828.

Bernstein, J. (2002) Polymorphism in Molecular Crystals. Clarendon Press, Oxford. 
Bernstein, J., Davey, R. J. and Henck, J.-O. (1999) Concomitant polymorphs. Angew. Chem. Int. Ed. 38: 3440-3461.

Buckton, G., Briggner, L.-E., Byström, K. and Darcy, P. (1994) The use of microcalorimetry in the study of changes in crystallinity induced during the processing of powders. Int. J. Pharm. 105: $125-135$.

Buckton, G. and Darcy, P. (1999) Assessment in crystalline powders - a review of analytical techniques and their application. Int. J. Pharm. 179: 141-158.

Bunn, C. W. (1948) Chemical Crystallography, An Introduction to Optical and X-ray Methods. Oxford University Press, London.

Chien, W.-M., Chandra, D., Helmy, A. K., Franklin, J. and Rawn, C. J. (2005) Experimental determination of $\mathrm{NH}_{4} \mathrm{NO}_{3}-\mathrm{KNO}_{3}$ binary phase diagram. $\mathrm{J}$ of Phase Equilibria and Diffusion 26: $115-123$.

Choi, C. S. and Prask, H. J. (1982) Single-crystal neutron diffraction study of ammonium nitrate phase III. Acta Cryst. B38: 2324-2328.

Choi, C. S. and Prask, H. J. (1983) The structure of ND4NO3 phase V by neutron powder diffraction. Acta Cryst. B39: 414-420.

Consalvo, D. and Stahl, W. (1998), The molecular structure of quinuclidine obtained by highpressure microwave spectroscopy. J. Mol. Struct. 447: 119-126.

Dellien, I. (1981) A DSC study of the phase transformations of ammonium nitrate. Thermochimica Acta 55: 181-191.

Desiraju, G. R. (1995) Supramolecular synthons in crystal engineering-a new organic synthesis. Angew. Chem. Int. Ed. Engl. 34: 2311-2327.

Desiraju, G. R. and Steiner, T. (1999) The Weak Hydrogen Bond in Structural Chemistry and Biology. Oxford University Press, Oxford.

Dunitz, J. D. and Bernstein, J. (1995) Disappearing Polymorphs. Acc. Chem. Res. 28: 193-200.

Dunitz, J. D. and Gavezzotti, A. (2005) Molecular recognition in organic crystals: directed intermolecular bonds or nonlocalised bonding? Angew. Chem. Int. Ed. 44: 1766-1787.

Ediger, M. D., Angell, C. A. and Nagel, S. R. (1996) Supercooled liquids and glasses. J. Phys. Chem. 100: 13200-13212.

Etter, M. C. (1990) Encoding and decoding hydrogen-bond patterns of organic compounds. Acc. Chem. Res. 23: 120-126.

Findlay, A. and Campbell, A. N. (1945) The Phase Rule and its Applications. 8 edn. Dover Publications, New York.

Giacovazzo, C. (ed.) (1992) Fundamentals of Crystallography. Oxford University Press, Oxford.

Giron, D. and Grant, D. J. W. (2002) Evaluation of solid-state properties of salts. In Handbook of Pharmaceutical Salts Properties, Selection and Use (eds Stahl, P. H. and Wermuth, C. G.). Verlag Helvetica Chimica Acta, Zürich, pp. 41-81.

Grant, D. J. W. (1999) Theory and origin of polymorphism. In Polymorphism in Pharmaceutical Solids (eds H. G. Brittain), Marcel Dekker, New York.

Grunenberg, A., Henck, J. O. and Siesler, H. W. (1996) Theoretical derivation and practical application of energy/temperature diagrams as an instrument in preformulation studies of polymorphic drug substances. Int. J. Pharm. 129: 147-158.

Harris, R. K., Hodgkinson, P., Larsson, T., Muruganantham, A., Ymén, I., Yufit, D. S. and Zorin, V. (2008) Characterization of polymorphs and solvates of terbutaline sulfate. Crystal Growth Des. 8: 80-90.

Hunter, C. A., Lawson, K. R., Perkins, J. and Urch, C. J. (2001) Aromatic interactions. J. Chem. Soc. Perkin Trans. 2: 651-669.

Jancic, S. J. and Grootscholten, P. A. M. (1984) Industrial Crystallization. Delft University Press, Delft. 
Jarring, K., Larsson, T., Stensland, B. and Ymén, I. (2006) Thermodynamic stability and crystal structures for polymorphs and solvates of formoterol fumarate. J. Pharm. Sci. 95: 1144-1161.

Jeffrey, G. A. (1997) An Introduction to Hydrogen Bonding. Oxford University Press, Oxford.

Kitaigorodsky, A. I. (1973) Molecular Crystals and Molecules. Academic Press, New York.

Klug, H. P. and Alexander, L. E. (1974) X-Ray Diffraction Procedures for Polycrystalline and Amorphous Materials. 2 edn. John Wiley \& Sons, Inc., New York.

Kubal, G., Sadler, P. J. and Tucker, A. (1994) pH induced structural changes in human serum apotransferrin. pKa-values for Histidine residues and $\mathrm{N}$-terminal amino group determined by 1H-NMR-spectroscopy. Europ. J. Biochem. 220: 781-787.

Leach, A. R. (2001) Molecular Modelling Principles and Applications. 2 edn. Pearson Education, Harlow.

Mason, C. W. (1983) Handbook of Chemical Microscopy, Volume 1. 4 edn. John Wiley \& Sons, Inc., New York.

Meenan, P. A., Andersen, S. R. and Klug, D. L. (2002) The influence of impurities and solvents on crystallization. In Handbook of Industrial Crystallization (eds A. S. Wyerson). 2 edn. Butterworth-Heinemann, Boston.

Mitzel N. W., Page E. M. and Rice D. A. (2000) Molecular structure of N-trimethylsilyaziridine in the gas phase. J Chem. Soc. Dalton Trans. 1491-1497.

Morissette, S. L., Almarsson, Ö., Peterson, M. L., Remenar, J. F., Read, M. J., Lemmo, A. V., Ellis, S., Cima, M. J. and Gardner, C. R. (2004) High-throughput crystallization: polymorphs, salts, co-crystals and solvates of pharmaceutical solids, Adv. Drug Del. Rev. 56: 275-300.

Morris, K. R. (1999) Structural Aspects of Hydrates and Solvates. In Polymorphism in Pharmaceutical Solids. Marcel Dekker, New York.

Moss, E. B. (1934) An apparatus for the determination of the dew point, Proc. Phys. Soc. 46: $450-458$.

Müller, A., Reuter, H. and Dillinger, S. (1995) Supramolecular inorganic chemistry: small guests in small and large hosts. Angew. Chem. Int. Ed. Engl. 34: 2328-2361.

Mullin, J. W. (2001) Crystallization. 4 edn. Butterworth-Heinemann, Boston.

Poonia, N. S. and Bajaj, A. V. (1979) Coordination chemistry of alkali and alkaline Earth cations. Chem. Rev. 79: 389-445.

Purdon, F. F. and Slater, V. W. (1946) Aqueous Solutions and the Phase Diagram. Edward Arnold \& Co., London.

Raghavan, V. and Cohen, M. (1975) Solid-state phase transformations. In Treatise on Solid State Chemistry. Volume 5. Changes of State (ed. N. B. Hannay). Plenum Press, New York.

Ramshaw, J. D. (1995) Fugacity and activity in a nutshell. J. Chem. Educ. 7: 601-603.

Rankin, D. W. H. and Robertson, H. E. (1995) Gas-Phase Molecular Structures Determined by Electron Diffraction. Spectroscopic Properties of Inorganic and Organometallic Compounds, (Specialist Periodical Report), Royal Society of Chemistry 28: 428-450.

Ricci, J. E. (1966) The Phase Rule and Heterogeneous Equilibrium, Dover Publications, New York.

Saleki-Gerhardt, A., Ahlnech, C. and Zografi, G. (1994) Assessment of disorder in crystalline solids. Int. J. Pharm. 101: 237-247.

Spitaleri, A., Hunter, C. A., McCabe, J., Packer, M. J. and Cockroft, S. L. (2004) A 1H NMR study of crystal nucleation in solution. Cryst. Eng. Comm. 6(80): 489-493.

Steiner, T. (2002) The hydrogen bond in the solid state. Angew. Chem. Int. Ed. 41: 48-76.

Tamari, S. and Aguilar-Chávez, A. (2004) Optimum design of the variable-volume gas pycnometer for determining the volume of solid particles. Meas. Sci. Technol. 15: 1146-1152.

Trask, A. V. and Jones, W. (2005) Crystal engineering of cocrystals by the solid state grinding approach. In Organic Solid State Reactions (ed. F. Toda). Springer-Verlag, Berlin. 
Vippagunta, S. R., Brittain, H. G. and Grant, D. J. W. (2001) Crystalline solids. Advanced Drug Delivery Reviews 48(1): 3-26.

Westdahl, M. (1996) Non-single Crystal X-ray Diffraction Methods. Thesis, Department of Structural Chemistry, Stockholm University.

Yalkowsky, S. H. and Banerjee, S. (1992) Aqueous Solubility Methods of Estimation for Organic Compounds. Marcel Dekker, New York.

Yu, L., Stephenson, G. A., Mitchell, C. A., Bunnell, C. A., Snorek, S. V., Bowyer, J. J., Borchardt, T. B., Stowell, J. G. and Byrn, S. R. (2000) Thermochemistry and Conformational Polymorphism of a Hexamorphic Crystal System. J. Am. Chem. Soc. 122: 585-591. 